FEDERAL

RESERVE

BANK of

ST. LOUIS
RESEARCH DIVISION

Working Paper Series

\title{
Dynamic Optimal Insurance and Lack of Commitment
}

\author{
Alexander K. Karaivanov \\ and \\ Fernando M. Martin
}

\author{
Working Paper 2011-029C \\ https://doi.org/10.20955/wp.2011.029
}

May 2014

\section{FEDERAL RESERVE BANK OF ST. LOUIS}

Research Division

P.O. Box 442

St. Louis, MO 63166

The views expressed are those of the individual authors and do not necessarily reflect official positions of the Federal Reserve Bank of St. Louis, the Federal Reserve System, or the Board of Governors.

Federal Reserve Bank of St. Louis Working Papers are preliminary materials circulated to stimulate discussion and critical comment. References in publications to Federal Reserve Bank of St. Louis Working Papers (other than an acknowledgment that the writer has had access to unpublished material) should be cleared with the author or authors. 


\title{
Dynamic Optimal Insurance and Lack of Commitment ${ }^{\text {ts }}$
}

\author{
Alexander K. Karaivanov ${ }^{\mathrm{a}}$, Fernando M. Martin ${ }^{\mathrm{a}, \mathrm{b}, *}$ \\ ${ }^{a}$ Simon Fraser University \\ ${ }^{b}$ Federal Reserve Bank of St. Louis
}

\begin{abstract}
We analyze dynamic risk-sharing contracts between profit-maximizing insurers and risk-averse agents who face idiosyncratic income uncertainty and can self-insure through savings. We study Markov-perfect insurance contracts in which neither party can commit beyond the current period. We show that the limited commitment assumption on the insurer's side is restrictive only when he is endowed with a rate of return advantage and the agent has sufficiently large initial assets. In such a case, the agent's consumption profile is distorted relative to the first-best. In a Markov-perfect equilibrium, the agent's asset holdings determine his outside option each period and are thus an integral part of insurance contracts, unlike when the insurer can commit long-term. Whether the parties can contract on the agent's savings decision affects the Markov-perfect contract as long as the insurer makes positive profits.
\end{abstract}

Keywords: optimal insurance, lack of commitment, Markov-perfect equilibrium, asset contractibility.

JEL classification: D11, E21.

\section{Introduction}

There are many situations in which people cannot commit to long-term contracts or are in fact legally constrained to short-term contracts. After some time, at least one party is usually free to rescind or request a change in the contract terms. For example, the majority of labor contracts are relatively short-term agreements: employees cannot legally commit to never quit and employers can terminate or change contract terms at a cost depending on the jurisdiction. Housing rental agreements are usually signed for no longer than a year with both sides able to terminate upon proper notice. Various insurance, TV, and phone service contracts have similar features. In this paper, we study one such situation assuming that the parties cannot commit to a contract longer than one period.

Consider a long-term interaction between a risk-averse agent facing idiosyncratic income risk and a riskneutral insurer. We analyze the best dynamic way for the insurer to extract profits from the agent when the insurer has a savings technology with a (weakly) superior rate of return than that of the agent. It is clear that if both parties could commit to an infinitely-long contract at time zero, the efficient outcome involves the insurer assuming control of the agent's initial assets, investing them in the superior savings technology, and appropriately compensating the agent with transfers over time. ${ }^{1}$ The insurer's ability to commit to compensate the agent in the future, after taking his assets, and the agent's commitment to not walk away from the contract ex-post, are key for this first-best arrangement to work. If such commitment is absent, then

\footnotetext{
${ }^{\sqrt{t}}$ We thank an anonymous referee, the Editor Marco Bassetto, D. Andolfatto, G. Camera, B. Camargo, M. Galenianos, M. Golosov, E. Green, D. Levine, M. Pycia, B. Ravikumar, V. Ríos-Rull, R. Rogerson, R. Townsend, and G. Ventura for various helpful comments and conversations. We also thank seminar and conference participants at the UBC-Kobe-SFU Workshop, Penn State, the Vienna Macro Workshop, the Federal Reserve Bank of Cleveland, the Midwest Macro Meeting, the Society for Economic Dynamics Meeting, the Far-Eastern Meeting of the Econometric Society, and the Canadian Macroeconomics Study Group. Karaivanov acknowledges the financial support of the Social Sciences and Humanities Research Council of Canada. The views expressed in this paper do not necessarily reflect official positions of the Federal Reserve Bank of St. Louis, the Federal Reserve System, or the Board of Governors.

* Corresponding author.

Email addresses: akaraiva@sfu.ca (Alexander K. Karaivanov), fernando.m.martin@stls.frb.org (Fernando M. Martin)

${ }^{1}$ For example, if the agent is more impatient than the insurer, it is optimal to front-load agent's consumption.
} 
the insurer faces a trade-off between rent extraction and production efficiency and hence, the agent is left with some of his assets to be carried at the inferior rate of return. Since assets determine the agent's outside option, their extraction can occur only gradually, distorting the optimal front-loading of consumption. In addition, the fact that the agent can walk away at some point of the contractual relationship limits how low his welfare can be driven in the long run, which further reduces total surplus.

The time profiles of the agent's consumption and the insurer's profits depend on the degree of the insurer's market power, which we allow to be anything between the two extremes of perfect competition and monopoly. Under perfect competition, expected profits are zero in net present value. If insurers lack commitment, free entry prevents them from earning positive expected period profits, which in turn implies they will not accept negative expected profits at any point in time. Thus, competitive insurers' expected profits are exactly zero and only period-by-period actuarially fair insurance is offered, with the agent's initial assets invested in the low-return technology until they are depleted. When the insurer has market power, the lack of commitment limits, but does not eliminate, his ability to extract profits efficiently.

To be more specific, suppose insurers can carry resources over time at the gross rate of return, $R$, while agents have access to their own savings technology with fixed exogenous gross return, $r$, where $r \leq R$. At the beginning of each period, an insurer can offer any new contract he likes and the agent is free to accept or go to his outside option. The agent's outside option depends on his current asset holdings and therefore evolves endogenously over time. ${ }^{2}$ We use the solution concept of Markov-perfect equilibrium (MPE), formally described in Maskin and Tirole (2001), to characterize dynamic insurance contracts in our setting. Markov-perfect equilibria capture the idea that only current, payoff-relevant variables (here, income realizations and agent's assets) affect the terms of equilibrium contracts and fit the notion of "bygones are bygones" - characteristics we consider natural in a lack of commitment environment.

Our main result is that the assumption that the insurer can commit only to a one-period contract, as opposed to an infinitely long contract, matters only if both of the following conditions hold: (i) the two parties' rates of return differ $(R>r)$ and (ii) the agent has sufficiently large initial assets. Intuitively, when the insurer's return is strictly higher than the agent's, there are gains from the insurer extracting the agent's assets up front and carrying them over time at the superior rate of return. However, this is possible only if the agent is appropriately compensated with promises of future consumption for which commitment by the insurer beyond the current period is indispensable. Lacking such commitment, we show that asset holdings by the agent become an integral part of Markov-perfect insurance contracts since they determine the value of the agent's outside option and hence the value of future transfers. In addition, the insurer's lack of commitment distorts the slope of the optimal consumption profile by introducing a "wedge" in the standard Euler equation whenever $r<R$.

In contrast, when either (i) the agent and the insurer face equal rates of return $(r=R)$ or (ii) the agent begins with zero or sufficiently low assets, we show that focusing on Markov-perfect insurance contracts is not restrictive at all: the same consumption time path and welfare are achieved in a one-sided commitment contract in which the insurer pre-commits to an infinitely long contract subject to a per period participation constraint by the agent. In the latter contract, assets and promised utility are interchangeable instruments that can be used by the insurer to ensure the agent stays on. As a result, a multiplicity of asset paths is possible. This multiplicity is avoided in an MPE where, as already mentioned, agent's asset holdings are a non-trivial feature of dynamic insurance which opens the way for calibrating versions of the model to data (see Karaivanov and Martin, 2012).

The agent's inability to commit beyond the current period, that is, being free to leave the contract at the beginning of each period, avoids the immiseration problems present in other papers in this literature. In the general case that we consider, when the product of the insurer's return $R$ and the agent's discount factor $\beta$ is less than 1, the optimal consumption path in the first best (full commitment to an infinitely long contract by both sides) is downward-sloping toward zero. Thus, eventually the agent will be worse off than his best alternative (autarky or any outside option that yields more than zero consumption forever). In contrast, in an MPE, consumption converges to a strictly positive value in finite time since the agent can always walk away. Hence, with the agent unable to commit beyond the current period, as long as his initial assets are positive, Markov-perfect insurance contracts always differ from the first-best contract, independent of the

\footnotetext{
${ }^{2}$ One possibility used by many authors is going to autarky forever, but we allow more general cases.
} 
parties' rates of return. ${ }^{3}$

Given that asset holdings by the agent are a key feature of Markov-perfect insurance contracts, we also study the role of asset contractibility by comparing the case of "contractible assets", when the insurer can fully control agent's savings, with the case of "non-contractible assets", in which the agent can privately decide on the amount of his savings but in a way observed by the insurer. In many situations, principals (governments, insurance companies, banks and so on) may have information about agents' assets but, for legal or other reasons, are unable to directly control agents' savings choices. ${ }^{4}$ In the dynamic insurance literature, allowing agents to accumulate assets typically yields one of three results, depending on the particular information and contractibility assumptions made: (i) assets play no role (when the insurer directly controls the agent's consumption); (ii) assets eliminate the insurer's ability to smooth the agent's consumption beyond selfinsurance (Allen, 1985; Cole and Kocherlakota, 2001); or (iii) the environment becomes highly intractable (Fernandes and Phelan, 2000; Doepke and Townsend, 2006). In contrast to the last case, we show that MPE insurance contracts result in simple dynamic programs with a single scalar state variable and avoid the curse of dimensionality when the principal cannot control the agent's savings.

We show that, in our setting, asset contractibility does not matter in the first best - the same optimal contract results. In contrast, in a Markov-perfect insurance contract, asset contractibility affects the contract terms in all cases except when there is a perfectly competitive insurance market with free entry by insurers. Intuitively, whenever the insurer has some market power (not necessarily as a monopolist) and can generate positive profits by insuring the agent, private asset accumulation by the agent gives him an instrument to counter the insurer by controlling his future outside option.

Unlike much of the previous literature on limited commitment (Thomas and Worrall, 1988, 1994; Ligon et al., 2002; Kocherlakota, 1996; Krueger and Uhlig, 2006; among many others), ${ }^{5}$ we assume that agents cannot renege after observing their income realization, that is, within the period. Reneging on an insurance contract after high income is realized is the primary reason full insurance may not be possible (at all, or in the short run) in those settings. In contrast, we obtain full insurance immediately and at all time. The lack of commitment friction we study is thus not about incentives to renege after obtaining windfall income, but about their ability to costlessly leave a contract before the period's uncertainty is resolved, should its terms become worse than one's outside option - not unlike in labor, health insurance, or similar contracts. The applications we have in mind are therefore not about agents acting opportunistically to obtain short-term gains from breaking a contract but about agents' freedom to continue or terminate a contractual relationship.

Our work is most closely related to that of Krueger and Uhlig (2006), Phelan (1995), and Kovrijnykh (2012). As in this paper, Krueger and Uhlig (2006) study dynamic risk-sharing between risk-neutral insurers and risk-averse agents who face idiosyncratic income uncertainty. Their model is most similar to our "onesided commitment" setting - insurers can fully commit to an infinitely long contract, while agents can renege in each period and move to another insurer without any cost or delay. A key difference is that agents in their paper can renege after observing the income realization. Thus, their main question is whether intratemporal insurance can be provided given the agent's commitment problem and potential for opportunistic behavior. Krueger and Uhlig show that the answer depends crucially on the relative size of the discount factors of the insurers and agents. Autarky, partial insurance, or even full insurance in the long run can be supported in equilibrium depending on the model parameters. Phelan (1995) studies dynamic risk-sharing with limited commitment in a competitive setting. His model is similar to ours in that the agent can renege on a contract before income is realized. Unlike here, Phelan assumes that agent's income is unobservable to the insurer and

\footnotetext{
${ }^{3}$ The only case in which these two contracts are equivalent is when the agent starts with zero assets and $\beta R=1$, since in this case the first-best contract is a repeated one-period contract.

${ }^{4}$ Hereby we differ from the literature on optimal contracting with hidden savings (Allen, 1985; Cole and Kocherlakota, 2001; among others) which assumes that the principal has no ability to monitor the agent's assets. On the technical side, our assumption of observable savings helps us avoid dynamic adverse selection and the possible failure of the revelation principle with lack of commitment (Bester and Strausz, 2001), while still preserving the intertemporal implications of savings noncontractibility.

${ }^{5}$ Thomas and Worrall (1988) characterize firm-worker contracts in which each party can deviate to autarky forever. Thomas and Worrall (1994) study limited commitment in a foreign direct investment problem with risk of expropriation. Ligon et al. (2002) analyze risk-sharing among agents who can revert to self-insurance through storage at any time. Kocherlakota (1996) studies risk-sharing between agents who cannot commit to not revert to autarky forever at any period. He analyzes the set of subgame-perfect equilibria and shows that, if agents are sufficiently patient, there is no efficiency loss associated with the inability to commit.
} 
studies the implications of this asymmetric information problem on the level of insurance provided. Setting $\beta r=1$, he shows that partial insurance can be achieved in equilibrium and that the long-run consumption distribution is non-degenerate.

In contrast to the Krueger-Uhlig and Phelan papers, the main question we study is not whether full insurance can or cannot be achieved (in our model it is always achieved) but the implications of lack of commitment across periods by agents and insurers on the time path of consumption relative to the first best and on private asset accumulation by the agent. Asset accumulation is not discussed by the above authors since the insurance contracts they study can be implemented through promised utility alone because of the insurers' full commitment ability. Additionally, our results cover the cases of non-competitive insurers and allow a general form for the agent's outside option.

Kovrijnykh (2012) studies a borrower-lender relationship with probabilistic enforcement. In essence, each period, after receiving a payment from the borrower, the lender may get an opportunity to renege on (change) his promised investment. Kovrijnykh finds that social welfare in this environment-regardless of whether contracts can be signed for one or infinitely many periods - can be lower than social welfare in an environment in which the lender cannot commit to honor the agreement within the period. As in the limited commitment literature cited above, Kovrijnykh thus studies opportunistic behavior (by the lender, in her case). She focuses on marginal increases in commitment power from no commitment to partial commitment by varying the contract enforcement probability. Our setting is different-we assume perfect enforcement within the period and interpret lack of commitment as the inability to bind oneself to actions beyond the current period. We show that under certain conditions (notably, equal rates of return on assets), an increase in the principal's commitment ability from one period to infinitely long yields equivalent welfare outcomes whereas otherwise full commitment dominates one-period commitment. ${ }^{6}$

More generally, our paper relates also to the work of Acemoglu et al. (2006) on optimal taxation with lack of commitment and the literature on "markets vs. mechanisms", for example, Bisin and Rampini (2006), Acemoglu et al. (2008), and Sleet and Yeltekin (2008) - who build various political economy models of governments unable to commit and analyze the extent to which they can improve on private-information constrained incomplete markets.

The rest of the paper is organized as follows. Section 2 presents the environment and characterizes the first-best contract with full commitment by both sides. Section 3 relaxes the commitment assumption, characterizes the properties of Markov-perfect contracts, and compares them to "one-sided commitment" contracts. Section 4 considers three natural extensions. First, we show that whether agent's assets are contractible (or not) affects the terms of Markov-perfect contracts, unlike in the first best. Second, we analyze the special case when insurance is provided in a perfectly competitive market with free entry. Third, we analyze the case when the agent and insurer bargain over the terms of the contract, which serves as an example of how to endogenize the agent's outside option which is taken as given in the previous sections. Section 5 concludes. All proofs are in the Appendix.

\section{The Model}

\subsection{Environment}

Consider a long-lived risk-averse agent who maximizes expected discounted utility from consumption. His period utility is $u(c)$, with $u_{c}(c)>0, u_{c c}(c)<0$, and $u$ satisfying Inada conditions. ${ }^{7}$ The agent discounts future utility by factor $\beta \in(0,1)$. He produces output $y$, which he can consume or save. Output is stochastic and equals $y^{i}$ with probability $\pi_{i}$, where $\pi_{i} \in(0,1)$ for all $i=1, \ldots, n$, with $n \geq 2$, and $\sum_{i=1}^{n} \pi_{i}=1$. Let $0 \leq y^{1}<\ldots<y^{n}$.

Since the agent is risk-averse and output is risky, the agent would like to smooth consumption over output states and time. Assets $a$ can be carried over time via a savings/storage technology with fixed gross return

\footnotetext{
${ }^{6}$ Sleet and Yeltekin (2006) consider lack of commitment by the principal in a dynamic private information economy. They show that the optimal allocations when the principal and agent have the same discount factor are equivalent to those in an economy with fully committed principals who discount the future less heavily than the agents. In contrast, allowing discount factors to differ or not, we demonstrate the importance of the two parties' rate of return on assets for the resulting outcomes with full vs. one-period commitment by the principal.

${ }^{7}$ Throughout the paper we use subscripts to denote partial derivatives and prime signs for next-period values.
} 
$r>0$. Let the set of feasible asset holdings be $\mathbb{A}=[0, \bar{a}]$, where $\bar{a} \in(0, \infty)$ and is chosen to be sufficiently large so that it is not restrictive (this is clarified below). If $y^{1}=0$, the lower bound on assets coincides with the "natural borrowing limit" (Aiyagari, 1994); if $y^{1}>0$, the lower bound on asset holdings may represent additional credit frictions in the environment. In terms of how dynamic contracts are affected by the frictions we study, our results are not affected by this particular assumption.

By standard arguments, the deterministic savings technology does not allow the agent to perfectly insure against the ( $n$-dimensional) output state randomness, thus there is scope for additional insurance. Suppose there exists a risk-neutral, profit-maximizing insurer (principal) who can provide such insurance. Throughout the paper, we assume that the insurer can costlessly observe output realizations $y^{i}$ and the agent's assets $a$ (see footnote 4).

The insurer discounts future profits at gross rate $R>1$. The parameter $R$ has either technological or preference interpretation. The special case $r=R$, for which several important results are derived, is interpreted as the insurer being able to carry resources intertemporally using the same technology as the agent. If instead $R=\beta^{-1}$, one can think of the contract sides as having the same discount factor - a standard assumption in the literature. In general, we allow $R$ to take any value in between these bounds. In addition, we assume $r<\beta^{-1}$ so that the agent's assets remain bounded, consistent with the definition of $\mathbb{A}$.

Assumption 1. $0<r \leq R \leq \beta^{-1}, r<\beta^{-1}$, and $R>1$.

\subsection{The agent's outside option}

Let the agent's outside option when contracting with the insurer be given by the function $B(a)$. Our results do no rely on the exact specifics of the agent's outside option beyond certain assumptions on $B$. By considering a general function $B(a)$ we can vary the split of the gains from trade between the insurer and the agent from the extreme case of a monopolistic insurer (in which all the surplus goes to the insurer), up to (but not including) the case of perfect competition in which all gains from trade go to the agent. The case of perfectly competitive (or "benevolent") insurer(s) is analyzed separately in Section 4.2 since it requires a different mathematical formulation.

We make the following assumptions on the agent's outside option.

Assumption 2. The agent's outside option function $B(a)$ has the following properties: (i) continuously differentiable, strictly increasing and strictly concave; (ii) $B(0)>\frac{u(0)}{1-\beta}$; (iii) $B(\bar{a})-\beta B(0)$ belongs to the range of $u$; and (iv) $B$ is such that the insurer can obtain positive surplus from providing insurance to the agent.

Part (i) of Assumption 2 lists technically desirable properties of the agent's outside option. Part (ii) ensures that the agent's inability to commit to a long-term contract (as analyzed in Sections 3 and 4) is relevant - that is, his outside option is such that the agent would eventually walk away from a contract that immiserates him. As shown below, parts (ii) and (iii) together guarantee that for any $a \in \mathbb{A}$ there exist positive consumption and admissible asset choices that satisfy the agent's participation constraint. Part (iv) implies that the insurer's participation constraint - zero profits - will not bind. Here, we are thinking of environments where very rich agents can still insure against fluctuations in income only imperfectly and where principals have sufficient market power to extract positive profits from an insurance agreement.

An example: autarky

One natural candidate for $B(a)$ is the agent's value function in autarky, i.e., $\Omega(a)$ determined by

$$
\Omega(a)=\max _{\left\{a^{i} \in \mathbb{A}\right\}_{i=1}^{n}} \sum_{i=1}^{n} \pi_{i}\left[u\left(r a+y^{i}-a^{i}\right)+\beta \Omega\left(a^{i}\right)\right] .
$$

Suppose $u(0)>-\infty$ or $y^{1}>0$. Then the autarky value function $\Omega(a)$ satisfies Assumption 2, parts (i), (ii) and (iv). By standard arguments (see Stokey, Lucas, and Prescott, 1989), our assumptions on $u$ ensure that $\Omega(a)$ satisfies (i). Part (ii) is satisfied since $y^{i}>0$ for all $i>1$ (i.e., expected consumption in autarky is positive at $a=0)$. Part (iv) holds by construction, as the agent cannot perfectly self-insure. For $B(a)=\Omega(a)$, Assumption 2(iii) is not necessary to guarantee existence of admissible consumption and asset choices that satisfy the participation constraint for all asset levels - existence follows directly from (1). 
The autarky (self-insurance) problem (1) is a standard "income fluctuation" problem studied, for instance, in Schechtman and Escudero (1977) or Aiyagari (1994), among many others. Its solution features:

(i) imperfect consumption smoothing $\left(c^{i}\right.$ differs across states with different $\left.y^{i}\right)$;

(ii) consumption, $c^{i}$ and asset choice, $a^{i}$ in each income state increasing in current assets, $a$;

(iii) asset contraction (negative savings) in the lowest income state(s) and asset accumulation (positive savings) for some range of asset holdings in the highest income state(s).

Since $r<\beta^{-1}$, the agent saves only to insure against consumption volatility. Clearly, then, an agent with more assets can do everything a poorer agent can, but the former is in a better position to self-insure against a long sequence of low outputs. The agent's inability to perfectly insure against income shocks implies that there is a demand for additional insurance.

In Section 4.3 we consider an alternative way to endogenize $B(a)$ by allowing the agent and principal to bargain over the surplus of the insurance contract.

\subsection{The first best}

We start by briefly characterizing the first-best contract in our setting, that is, the optimal insurance contract when both parties can fully commit to an agreement signed at time zero. This is the natural benchmark against which we analyze the role of frictions - limited commitment and asset non-contractibility -in dynamic insurance. The first-best contract specifies state-contingent transfers/consumption and savings decisions. The only restrictions on it are the ex-ante participation constraints of the contracting parties. By our assumptions on $B(a)$ we impose only the agent's participation constraint.

In the first best, a long-term binding agreement is signed at the initial date specifying the complete path of history-contingent outcomes for all future periods and states. Thereafter, the timeline within each time period is as follows. Output is realized in the beginning of the period. Then transfers from/to the agent take place. Finally, the agent consumes and saves the contracted amounts.

Note that due to the timing of events, if allowed to, the agent may, in principle, wish to deviate from the specified contract by varying his asset holdings and thus, his consumption. Below, we show that this is not the case with full commitment. That is, the first-best contract remains incentive-feasible even when the agent's savings are non-contractible - who controls asset accumulation does not matter with full commitment. In contrast, in Section 4.1 we show that, in the absence of commitment, asset (non-)contractibility does affect the resulting dynamic insurance contract.

Proposition 1. The first-best insurance contract has the following properties:

(i) no asset accumulation by the agent, $a_{t}=0$ for all $t>0$, is optimal;

(ii) equal consumption across output states in all periods, $c_{t}^{i}=c_{t}$ for all $i=1, \ldots, n$ and all $t \geq 0$;

(iii) decreasing consumption profile (strictly, if $\beta R<1$ ), $c_{t} \geq c_{t+1}$ satisfying $u_{c}\left(c_{t}\right)=\beta R u_{c}\left(c_{t+1}\right)$ for $t \geq 0$ at an interior solution;

(iv) remains incentive-compatible when agent's savings are non-contractible.

The results in parts (ii) and (iii) are standard. Since there is no private information or other incentive problems, the first-best contract gives the risk-averse agent equal consumption across output states in all periods. If the parties discount at the same rate (the special case $R=\beta^{-1}$ ), the first-best consumption profile is constant over time, while if $R<\beta^{-1}$, the agent's consumption is strictly decreasing over time toward zero.

Regarding asset accumulation by the agent in the first best, Proposition 1(i) shows that agent's assets can be (must be, if $r<R$ ) optimally extracted upfront, at $t=0$ by the insurer and set to zero thereafter. In contrast, as we show in the next section, with lack of commitment, private asset accumulation by the agent is an integral part of dynamic insurance contracts. In part (iv) we show that asset contractibility does not affect the optimal contract: with full commitment, the first best can still be implemented when the principal has no control over the agent's observable assets since it remains incentive-compatible. The intuition is that the agent has no incentive to self-insure or protect himself against changes in the contract terms by saving privately since he faces full insurance in an infinitely-long contract. 
Let $\bar{y} \equiv \sum_{i=1}^{n} \pi_{i} y^{i}>0$ be expected output and $c_{t} \equiv r a_{t}+\tau_{t}-a_{t+1}$ be period- $t$ consumption implied by a contract offering $\left\{\tau_{t}, a_{t+1}\right\}_{t=0}^{\infty}$. By Proposition 1 (see the proof in the Appendix for full details) we can write the first-best contracting problem as a two-stage problem. In the first stage, the insurer solves a static problem in which he extracts the agent's initial assets $a_{0}$ and promises lifetime discounted utility $w$ from the next period on, subject to the agent's time-zero participation constraint:

$$
\begin{aligned}
& \Pi^{F B}\left(a_{0}\right)=\max _{c_{0}, w} r a_{0}+\bar{y}-c_{0}+R^{-1} \tilde{\Pi}^{F B}(w) \\
& \text { subject to } u\left(c_{0}\right)+\beta w-B\left(a_{0}\right) \geq 0
\end{aligned}
$$

where the function $\tilde{\Pi}^{F B}(w)$ solves the following, second-stage dynamic program of maximizing the insurer's profits subject to a promise-keeping constraint:

$$
\begin{aligned}
& \tilde{\Pi}^{F B}(w)=\max _{c, w^{\prime}} \bar{y}-c+R^{-1} \tilde{\Pi}^{F B}\left(w^{\prime}\right) \\
& \text { subject to } u(c)+\beta w^{\prime}-w=0 .
\end{aligned}
$$

In the first best, both the principal and the agent fully commit to follow the agreement signed at time zero. While it is the most efficient ex-ante, the first-best contract in our setting generically has the property that, ex-post, both parties would wish they could renege on it. For the agent, the reason is that his participation constraint is imposed only ex-ante. In the general case $R<\beta^{-1}$, the strictly decreasing consumption time path in the first-best contract implies that at some point in time the agent would be better off by switching to his outside option with zero assets, $B(0)$. For the insurer, recall that when the agent's initial assets $a_{0}$ are extracted at the start of the first-best contract, in compensation the agent is issued "credit" in the form of promised utility. Thus, even if the agent were committed to remain in the contract forever, as long as $a_{0}>0$, the insurer would like to renege on his past promises (worth $B\left(a_{0}\right)$ in total, which is strictly larger than $B(0))$ and extract the most surplus from the current period onward. ${ }^{8}$

\section{Lack of Commitment}

\subsection{Markov-perfect insurance contracts}

Motivated by the examples in the introduction, assume that neither the principal nor the agent can bind themselves to a contract extending beyond the current period. That is, only one-period contracts can be enforced. Note that we assume no penalties for failing to reach an agreement.

In our environment, individual punishment strategies such as, for example, the threat to never again sign a contract, are not credible - given our assumptions, at the beginning of each period there are always gains from insurance on the table. We adopt the solution concept of Markov-perfect equilibrium (MPE) (Maskin and Tirole, 2001) and characterize the best possible dynamic insurance contracts with (double-sided) lack of commitment that are solely functions of fundamentals: beginning-of-period assets and current output realizations. Then, in Section 3.3 we show conditions under which the resulting equilibrium is equivalent to an infinitely long contract in which only the insurer can fully commit.

In an MPE, the insurer offers the agent a contract for the current period, $\left\{\tau^{i}, a^{i}\right\}_{i=1}^{n}$, consisting of outputcontingent transfers and end-of-period asset holdings, taking as given anticipated future interactions between himself and the agent. Specifically, given the agent's current assets $a$, the problem of the insurer today is to choose $\left\{\tau^{i}, a^{i}\right\}_{i=1}^{n}$, taking as given future decision rules $\left\{\mathcal{T}^{i}(a), \mathcal{A}^{i}(a)\right\}_{i=1}^{n}$ for transfers and assets, which induce profits $\Pi(a)$ and agent's continuation value $v(a)$.

For now, we maintain the assumption that the agent's assets are contractible, that is, the insurer can specify and control the agent's consumption and savings for the period (the case of non-contractible assets is analyzed in Section 4.1). Each period, the agent needs to decide whether to accept the currently offered contract. Given future interactions between the agent and the insurer that induce profits $\Pi(a)$ (to be defined

\footnotetext{
${ }^{8}$ In the special case $R=\beta^{-1}$, the agent receives future discounted utility of $B\left(a_{0}\right)$ each period. After assets are extracted, the insurer would still like to renege on the promised utility $B\left(a_{0}\right)$ and drive the agent to his new outside option $B(0)$. Only in the knife-edge case, when both $R=\beta^{-1}$ and $a_{0}=0$ hold, does the first-best contract become a repeated static contract and as such, renegotiation-proof.
} 
below), a continuation value $v(a)$ (to be determined below), and an outside option given by $B(a)$, the problem of the insurer facing an agent with current assets $a$ is:

$$
\max _{\left\{\tau^{i}, a^{i} \in \mathbb{A}\right\}_{i=1}^{n}} \sum_{i=1}^{n} \pi_{i}\left[y^{i}-\tau^{i}+R^{-1} \Pi\left(a^{i}\right)\right]
$$

subject to the agent's participation constraint

$$
\sum_{i=1}^{n} \pi_{i}\left[u\left(r a+\tau^{i}-a^{i}\right)+\beta v\left(a^{i}\right)\right]-B(a) \geq 0 .
$$

Note the difference between the time-zero participation constraint in the first-best problem (2) and the period-by-period participation constraint (5).

The assumed strict concavity of $u$ implies that full insurance is optimal; that is, $c^{i} \equiv r a+\tau^{i}-a^{i}$ are equalized across the output states $i=1, \ldots, n$ (formally, this can be easily shown by taking the first-order conditions of problem (4)-(5) with respect to $\left.\tau^{i}\right)$. Intuitively, in the absence of incentive provision concerns, it is inefficient to have the risk-averse agent bear any risk across states. What is key for this result is that in our setting the parties cannot renege after output is realized. Note again the contrast with other papers on optimal insurance with limited commitment (e.g., Kocherlakota, 1996; Ligon, Thomas, and Worrall, 2002; Krueger and Uhlig, 2006), where agents can renege on the insurance scheme after observing the output and so only partial insurance may be sustained. In addition, the first-order conditions with respect to $a^{i}$ of problem (4)-(5) are symmetric across output states. Assuming a symmetric solution (we show existence below), we thus have $c^{i}=c^{j}=c, a^{i}=a^{j}=a^{\prime}$, and $\tau^{i}=\tau^{j}=\tau$ for all $i, j=1, \ldots, n$.

Lemma 1. The agent's participation constraint, (5) is satisfied with equality and $v(a)=B(a)$ for all $a \in \mathbb{A}$.

Intuitively, today's principal leaves no surplus to the agent regardless of how surplus is distributed in the future. In particular, if for some reason the insurer tomorrow left the agent better off than with his outside option, because of lack of commitment, the insurer today would still find it optimal to extract all surplus. As a result, the agent is always exactly as well off as with his outside option.

Using these results, we formally define a Markov-perfect equilibrium and a Markov-perfect contract in our setting as follows.

Definition 1. (i) Given B(a) satisfying Assumption 2, a Markov-perfect equilibrium (MPE) is a set of functions $\{\mathcal{T}, \mathcal{A}, \Pi\}: \mathbb{A} \rightarrow \mathbb{R} \times \mathbb{A} \times \mathbb{R}_{+}$defined such that, for all a $\in \mathbb{A}$ :

$$
\{\mathcal{T}(a), \mathcal{A}(a)\}=\underset{\tau, a^{\prime} \in \mathbb{A}}{\operatorname{argmax}} \bar{y}-\tau+R^{-1} \Pi\left(a^{\prime}\right)
$$

subject to

$$
u\left(r a+\tau-a^{\prime}\right)+\beta B\left(a^{\prime}\right)-B(a)=0
$$

and

$$
\Pi(a)=\bar{y}-\mathcal{T}(a)+R^{-1} \Pi(\mathcal{A}(a)) .
$$

(ii) For any $a \in \mathbb{A}$, the Markov-perfect contract implied by an MPE, as defined in (i), is the transfer and savings pair: $\left\{\tau=\mathcal{T}(a), a^{\prime}=\mathcal{A}(a)\right\}$.

To simplify the exposition, let $\mathcal{C}(a) \equiv r a+\mathcal{T}(a)-\mathcal{A}(a)$ denote the consumption function in an MPE and let $c \equiv r a+\tau-a^{\prime}$ denote the consumption in a Markov-perfect contract $\left\{\tau, a^{\prime}\right\}$ given any $a \in \mathbb{A}$. Using Definition 1, rewrite the Markov-perfect contracting problem in a mathematically equivalent way as

$$
\Pi(a)=\max _{c, a^{\prime} \in \mathbb{A}} \bar{y}+r a-c-a^{\prime}+R^{-1} \Pi\left(a^{\prime}\right)
$$

subject to

$$
u(c)+\beta B\left(a^{\prime}\right)-B(a)=0 .
$$


The insurer takes the outside option function $B(a)$ as given - he cannot control its value except via the agent's assets; for example, as in the case of autarky when $B(a)=\Omega(a)$ for all $a \in \mathbb{A} .{ }^{9}$ To solve problem (6)-(7), we need to find a fixed-point in the function $\Pi$. Assumption 2 ensures that the constraint set is non-empty; that is, $\forall a \in \mathbb{A}$ there exist $c>0$ and $a^{\prime} \in \mathbb{A}$ satisfying (7) (see footnote 17 in the Appendix). Since the set $\mathbb{A}$ is compact, standard contraction mapping arguments ensure the existence and continuity of the value function $\Pi$ (Stokey, Lucas, and Prescott, hereafter, SLP, 1989; see also Krueger and Uhlig, 2006). More specifically, as in Krueger and Uhlig (2006), using the fact that $u$ and $B$ are strictly increasing and changing variables to $z \equiv B(a)$, we can rewrite problem (6)-(7) as

$$
\Pi^{*}(z)=\max _{z^{\prime}} \bar{y}+F\left(z, z^{\prime}\right)+R^{-1} \Pi^{*}\left(z^{\prime}\right)
$$

where $F\left(z, z^{\prime}\right) \equiv r B^{-1}(z)-u^{-1}\left(z-\beta z^{\prime}\right)-B^{-1}\left(z^{\prime}\right)$ for $z, z^{\prime} \in[B(0), B(\bar{a})]$, and $z-\beta z^{\prime}$ in the range of $u$. It is easy to verify that SLP Assumptions 4.3, 4.4 and 4.8 are satisfied. By SLP Theorem 4.6, this implies uniqueness of the fixed point $\Pi$. A sufficient condition for continuous and single-valued policy $z^{\prime}$ in problem (8) and hence continuous functions $\mathcal{T}, \mathcal{C}$ and $\mathcal{A}$ in the original MPE problem, is $F\left(z, z^{\prime}\right)$ to be strictly concave (see SLP, 1989, Assumption 4.7 and Theorem 4.8). Assume $u$ and $B$ are such that this condition holds. ${ }^{10}$ Strict concavity of $F$ thus implies the existence and uniqueness of the MPE as described in Definition 1. Next, by the differentiability of $u$ and $B, F$ is continuously differentiable on the interior of its domain. This implies that $\Pi^{*}$ is differentiable on the interior of its domain and hence $\Pi$ is differentiable on the interior of $\mathbb{A}$ (see SLP, 1989, Assumption 4.9 and Theorem 4.11). Figure 1 below illustrates a numerical example of value and policy functions satisfying these properties.

The first-order conditions of problem (6)-(7) with respect to $c$ and $a^{\prime}$ are

$$
\begin{array}{r}
-1+\lambda u_{c}(c)=0 \\
-1+R^{-1} \Pi_{a}\left(a^{\prime}\right)+\lambda \beta B_{a}\left(a^{\prime}\right)+\zeta=0,
\end{array}
$$

where $\lambda$ and $\zeta$ are the Lagrange multipliers associated with the participation constraint and the nonnegativity constraint on future assets, respectively. ${ }^{11}$

Note that $\lambda=1 / u_{c}>0$. The envelope condition implies

$$
\Pi_{a}(a)=r-\frac{B_{a}(a)}{u_{c}(c)} .
$$

Substituting (9) and $\lambda=1 / u_{c}$ into the first-order condition for $a^{\prime}$, the equations characterizing a Markovperfect insurance contract for any $a \in \mathbb{A}$ are the participation constraint (7) and

$$
r-R+B_{a}\left(a^{\prime}\right)\left[\frac{\beta R}{u_{c}(c)}-\frac{1}{u_{c}\left(c^{\prime}\right)}\right]+\zeta R=0 .
$$

Proposition 2. A Markov-perfect equilibrium has the following properties:

(i) assets profile: $\exists \hat{a} \in(0, \bar{a}]$ such that $\mathcal{A}(a)=0$ for all $a \in[0, \hat{a}]$ and $0<\mathcal{A}(a)<a$, with $\mathcal{A}(a)$ strictly increasing for all $a \in \mathbb{A} \backslash[0, \hat{a}]$;

(ii) consumption profile: $\mathcal{C}(\mathcal{A}(a))<\mathcal{C}(a)$ for all $a \in(0, \bar{a}]$, with $\mathcal{C}(a)$ strictly increasing for all $a \in \mathbb{A}$;

(iii) zero assets in finite time and positive long-run consumption: $\exists T<\infty$ such that $a_{T} \equiv \mathcal{A}\left(\mathcal{A}\left(\ldots \mathcal{A}\left(a_{0}\right) \ldots\right)\right)(T-$ times $)=0$, for all $a_{0} \in \mathbb{A} ;$ and $\mathcal{C}(0)>0$.

\footnotetext{
${ }^{9}$ We study examples of endogenously generated outside options in Section 4.3 .

${ }^{10}$ For example, take some strictly increasing, strictly concave, and unbounded above $u$ and suppose $B(a)=b_{0}+a^{\nu} / b_{1}$, where $b_{0}>u(0) /(1-\beta), b_{1}>0$, and $\nu<1$. As $\nu \rightarrow 1$, we have $F\left(z, z^{\prime}\right) \rightarrow \kappa+b_{1}\left(r z-z^{\prime}\right)-u^{-1}\left(z-\beta z^{\prime}\right)$, where $\kappa$ is a constant. Since $u$ is strictly concave, $-u^{-1}$ is strictly concave as well. For any $\lambda \in(0,1)$ and any admissible $\left(z_{1}, z_{1}^{\prime}\right)$ and $\left(z_{2}, z_{2}^{\prime}\right)$, let $z_{\lambda} \equiv \lambda z_{1}+(1-\lambda) z_{2}$ and $z_{\lambda}^{\prime} \equiv \lambda z_{1}^{\prime}+(1-\lambda) z_{2}^{\prime}$. Then we have that $F\left(z_{\lambda}, z_{\lambda}^{\prime}\right)=\kappa+b_{1}\left(r z_{\lambda}-z_{\lambda}^{\prime}\right)-u^{-1}\left(z_{\lambda}-\beta z_{\lambda}^{\prime}\right)=\kappa+b_{1}\left(r z_{\lambda}-\right.$ $\left.z_{\lambda}^{\prime}\right)-u^{-1}\left(\lambda\left(z_{1}-\beta z_{1}^{\prime}\right)+(1-\lambda)\left(z_{2}-\beta z_{2}^{\prime}\right)\right)>\kappa+\lambda b_{1}\left(r z_{1}-z_{1}^{\prime}\right)+(1-\lambda) b_{1}\left(r z_{2}-z_{2}^{\prime}\right)-\lambda u^{-1}\left(z_{1}-\beta z_{1}^{\prime}\right)-(1-\lambda) u^{-1}\left(z_{2}-\beta z_{2}^{\prime}\right)=$ $\lambda F\left(z_{1}, z_{1}^{\prime}\right)+(1-\lambda) F\left(z_{2}, z_{2}^{\prime}\right)$. Therefore, $F$ is strictly concave.

${ }^{11}$ As we show in Proposition 2, in an MPE, $a^{\prime} \geq 0$ binds for some asset levels, while $a^{\prime} \leq \bar{a}$ never binds.
} 
Typically, the savings function in an $\operatorname{MPE}, \mathcal{A}(a)$, is equal to zero between zero assets and a critical value $\hat{a}>0$, and is strictly increasing but stays below the 45-degree line for higher asset levels. It is possible, however, that the $u$ and $B$ functions and the set $\mathbb{A}$ are such that $\mathcal{A}(a)=0$ for all $a \in \mathbb{A}$ - that is, it may be that there is no interior solution for savings for any asset level in the admissible set. We can use the results from Proposition 2 to derive a condition ensuring that an interior solution exists for sufficiently high asset levels. At the critical point $\hat{a}$, by definition, the optimal choice is zero assets and $\zeta=0$. Consumption at $\hat{a}$ and in the following period can be derived from the agent's participation constraint: $u(\mathcal{C}(\hat{a}))=B(\hat{a})-\beta B(0)$ and $u(\mathcal{C}(0))=(1-\beta) B(0)$. Note that Assumption 2 implies $\mathcal{C}(0)>0$ and $\mathcal{C}(\hat{a})>0$. Thus, we can use (10) to solve for $\hat{a}$ :

$$
r-R+B_{a}(0)\left[\frac{\beta R}{u_{c}\left(u^{-1}(B(\hat{a})-\beta B(0))\right)}-\frac{1}{u_{c}\left(u^{-1}((1-\beta) B(0))\right)}\right]=0 .
$$

Hence, to ensure that $\hat{a} \in(0, \bar{a})$ we need to pick $u$ and $B$ such that there exists $\hat{a}>0$ which solves (11) and next pick some $\bar{a}>\hat{a}$ and define $\mathbb{A}=[0, \bar{a}]$.

Figure 1 displays a computed example of an MPE, which illustrates the results in Proposition 2. The MPE is compared with the autarky solution, problem (1). Displayed are transfers, savings, consumption, and net present value profits in the MPE, each as function of assets.

Figure 1: Markov-perfect equilibrium

[Figure 1 about here]

Note: Solid lines correspond to variables in an MPE; the dashed lines correspond to variables in autarky. There are two output states, labeled $y^{L}$ and $y^{H}$. Asset accumulation and consumption in autarky are given by $a^{H}, a^{L}, c^{H}$ and $c^{L}$. The assumed parameterization is $u(c)=\ln c, \beta=0.93 ; r=1.06 ; R=1.07 ; y^{1}=0.1, y^{2}=0.3$, and $\pi^{1}=0.5$.

\subsection{Comparison with the first best}

Compare the results of Proposition 2 characterizing Markov-perfect insurance contracts with the properties of the first-best contract in Proposition 1. First, the role of asset accumulation is very different. Intuitively, while in the first best it is (weakly) optimal to extract all agent assets at time zero, in an MPE, without commitment by the insurer, any positive initial wealth can be only gradually reduced over time as dictated by the per-period participation constraints.

Second, the insurer's lack of commitment distorts the slope of the consumption profile. In the first best (see Proposition 1), the consumption profile satisfies the standard Euler equation, $u_{c}(c)=\beta R u_{c}\left(c^{\prime}\right)$. In contrast, in our MPE we can rewrite (10) at an interior solution as the following modified Euler equation:

$$
u_{c}(c)=\beta R u_{c}\left(c^{\prime}\right)\left[1-\frac{(R-r)}{R} \frac{u_{c}(c)}{\beta B_{a}\left(a^{\prime}\right)}\right] .
$$

The non-positive wedge, $-\frac{(R-r)}{R} \frac{u_{c}(c)}{\beta B_{a}\left(a^{\prime}\right)}$, reflects the inefficiency introduced by the principal's lack of commitment. In the first-best contract, the insurer extracts all agent assets at $t=0$ and invests them in the more productive asset accumulation technology with return $R$ (this is without loss of generality when $r=R$ ). In an MPE, since the principal lacks commitment, he cannot compensate the agent for such immediate asset extraction with promised utility (or anything else); thus, he can only run down the agent's savings gradually, respecting the participation constraint. This distorts efficiency whenever $R>r$ since assets are carried over time at the low return $r$. In the resulting Euler equation wedge in (12), the term $\frac{u_{c}(c)}{\beta B_{a}\left(a^{\prime}\right)}$ states the trade-off faced by the principal: each additional unit of assets he would like to extract lowers the agent's future outside option by $\beta B_{a}$ and hence, needs to be compensated now with additional consumption worth $u_{c}$. Naturally, the size of the distortion arising from not being able to extract assets optimally is proportional to the difference between the insurer's and agent's intertemporal rates of return, $R-r$.

In terms of the consumption time profile, in the first best, consumption follows $u_{c}(c)=\beta R u_{c}\left(c^{\prime}\right)$. Thus, if $R \beta<1$, first-best consumption decreases over time and converges to zero, whereas if $R \beta=1$, first-best consumption is constant over time and equals $u^{-1}\left((1-\beta) B\left(a_{0}\right)>0\right.$. In contrast, consumption in an MPE 
follows (12), which implies $u_{c}(c)<\beta R u_{c}\left(c^{\prime}\right)$ if $r<R \leq \beta^{-1}$ or $u_{c}(c)=\beta R u_{c}\left(c^{\prime}\right)$ if $r=R<\beta^{-1}$. Therefore, in both cases $u_{c}(c)<u_{c}\left(c^{\prime}\right)$, i.e., MPE consumption decreases over time until the agent's assets are depleted. When assets reach zero, MPE consumption $\mathcal{C}(0)$ is equal to $u^{-1}\left((1-\beta) B(0)>0\right.$ from that point on. ${ }^{12}$ These results imply that the Markov-perfect and first-best contracts coincide only when $R \beta=1$ and $a_{0}=0$ (see also footnote 8 ).

\subsection{Markov-perfect insurance vs. one-sided commitment}

To clarify further the role of commitment by each side in the dynamic insurance problem, we compare and contrast our Markov-perfect contracts, as defined and characterized above, with the situation in which only the insurer has long-term commitment ability. More precisely, consider a one-sided commitment setting in which, as in the first best (Section 2.3), the insurer is able to commit to an infinite sequence of statecontingent transfers at time zero while, as in Section 3.1, the agent is allowed to walk away from the contract at the beginning of each period before output is realized and cannot renege on the contract for the period if he decides to stay on. The latter implies that, as in an MPE, the agent's participation constraint must be satisfied in every period.

By the same arguments as in Section 2.3, full insurance obtains. Also, as in Proposition 1, it is optimal for the principal to extract the agent's assets in the initial period. Thus, as we did for the first best in (2)-(3), we can write the insurer's problem with one-sided commitment as a two-stage problem. In the first stage, the insurer solves a static problem in which he extracts the agent's initial assets, $a_{0}$, and promises in exchange lifetime utility $w$ from next period onward, subject to the agent's limited-commitment constraint $w \geq B(0)$. In the second stage, the optimal consumption and promised utility are chosen respecting a promise-keeping constraint to deliver the promise $w$.

As in Phelan (1995) or Krueger and Uhlig (2006), suppose that the feasible values for promised utility in the one-sided commitment setting belong to the set $\left[B(0), \bar{w}\left(a_{0}\right)\right]$ with the upper bound $\bar{w}\left(a_{0}\right)$ chosen sufficiently large, (e.g., the level of discounted future utility that requires at least as many resources to be delivered as available to the insurer in net present value). ${ }^{13}$ Similar to Phelan (1995), for the moment we treat $\bar{w}\left(a_{0}\right)$ as exogenously given. However, in the proof of Proposition 3 below we show that we can set $\bar{w}\left(a_{0}\right)=B\left(a_{0}\right)$ without restricting the insurer's optimal choices in the one-sided commitment problem-that is, all optimally chosen promised utilities belong to $\left[B(0), B\left(a_{0}\right)\right]$.

For this part of the paper we assume, in addition to our previous assumptions,

Assumption 3. $B(\bar{a}) \geq \bar{w}\left(a_{0}\right)$ for all $a_{0} \in \mathbb{A}$.

This assumption ensures that the range of the function $B$ is sufficiently wide so that, for any initial $a_{0}$, any promised utility chosen in the one-sided commitment problem can be mapped into an appropriate asset choice and corresponding outside option value in the MPE problem (see Proposition 3 and its proof for the details).

To formulate the insurer's problem with one-sided commitment, define the choice set for promised utility as $W \equiv[B(0), B(\bar{a})]$, which by Assumption 3 is not restrictive for any $a_{0}$. We will show that, for $r=R$ or $a_{0} \in[0, \hat{a}]$, the optimal one-sided commitment contract solving problem (13)-(14) below and our MPE imply identical consumption sequences over time starting from the same $a_{0}$ - that is, there is a one-to-one mapping between the path of optimally chosen promised utilities in the one-sided contract starting from $a_{0}$ and the path of optimally chosen asset levels in MPE starting from the same initial assets $a_{0}$.

The one-sided commitment problem is

$$
\Pi^{O S}\left(a_{0}\right)=\max _{c_{0}, w \in W} \bar{y}+r a_{0}-c_{0}+R^{-1} \tilde{\Pi}^{O S}(w)
$$

subject to

$$
u\left(c_{0}\right)+\beta w-B\left(a_{0}\right)=0
$$

\footnotetext{
${ }^{12}$ Note that positive long-run consumption follows from Assumption 2(ii), but this result does not rely on the assumed borrowing constraint. Even if the agent were allowed to borrow up to the natural borrowing limit (a case we subsume if $\left.y^{1}=0\right)$, his outside option $B(0)$ dominates the present value of zero consumption forever.

${ }^{13}$ Clearly, no levels of promised utility $w<B(0)$ are relevant choices for the insurer, since the agent would always walk away to his outside option.
} 
The limited commitment constraint $w \geq B(0)$ is imposed by the lower bound of the set $W$. The function $\tilde{\Pi}^{O S}(w)$ is the solution to the following second-stage dynamic program defined for any $w \in W$ chosen in (13):

$$
\tilde{\Pi}^{O S}(w)=\max _{c, w^{\prime} \in W} \bar{y}-c+R^{-1} \tilde{\Pi}^{O S}\left(w^{\prime}\right)
$$

subject to

$$
u(c)+\beta w^{\prime}-w=0 .
$$

The key differences with the first-best contract solving (2)-(3) are the additional inequality constraints on future promised utility at each stage: $w-B(0) \geq 0$ and $w^{\prime}-B(0) \geq 0$ implied in the restrictions $w \in W$ and $w^{\prime} \in W$, respectively. These constraints embody the agent's inability to commit to not renege on the contract at the beginning of a period and obtain his outside option $B(0)$. In contrast, in the first best, promised utility can fall below $B(0)$ in the long run (e.g., if $\beta R<1$, when consumption converges to zero; see Assumption 2).

\section{Proposition 3. Markov-perfect vs. one-sided commitment contracts.}

(i) If $r=R$ or $a_{0} \in[0, \hat{a}]$, where $\hat{a}$ is defined in Proposition 2(i), then, from the same initial asset level $a_{0} \in \mathbb{A}$, an MPE where the insurer solves problem (6)-(7) implies identical consumption sequence $\left\{c_{t}\right\}_{t=0}^{\infty}$ and time-zero discounted profits as the one-sided commitment contract defined by (13)-(14).

(ii) If $r<R$ and $a_{0}>\hat{a}$, where $\hat{a}$ is defined in Proposition 2(i), then, from the same initial asset level, Markov-perfect insurance contracts differ from one-sided commitment contracts in the implied consumption sequence $\left\{c_{t}\right\}_{t=0}^{\infty}$ and profits. Specifically,

(a) time-zero discounted profits are strictly higher with one-sided commitment;

(b) the agent's consumption time profile with one-sided commitment satisfies $u_{c}(c)=\beta R u_{c}\left(c^{\prime}\right)$ and so differs from that in an MPE which satisfies (12) but, as long as $R \beta<1$, long-run consumption is the same in both settings.

The results in Proposition 3 add to the previous discussion in Section 3.2, further clarifying the role of commitment by the insurer. Part (i) establishes that, when the insurer and agent face the same rate of return on carrying assets over time, our Markov-perfect insurance contracts from Section 3.1 achieve the same consumption and profit time paths as optimal dynamic insurance contracts in which the insurer can commit to a long-term ex-ante contract but the agent cannot. Intuitively, when both parties can carry assets at the same rate of return, promised utility and contractible assets are fully interchangeable from the point of view of the insurer - everything that can be implemented with one of these two instruments can be exactly replicated with the other.

However, Proposition 3, part (ii) shows that one-sided commitment and Markov-perfect contracts do differ when $r<R$ and $a_{0}>\hat{a}$ (the interior solution case). One-sided commitment contracts generate more surplus than Markov-perfect contracts starting from the same initial asset level. Intuitively, the principal's long-term commitment ability in the one-sided setting enables him to extract agent's assets in the initial period in exchange for promised utility and then use his superior rate of return to generate higher total surplus. Obviously, for any gains to be realized from this asset extraction, the agent's assets must be positive in an MPE for some $t>0$. The agent agrees to hand over his assets at $t=0$ in the one-sided commitment setting because he knows that the insurer cannot renege on his promise to compensate him with future transfers. The rate of return differential causes the consumption time profiles to differ but, as long as $R \beta<1$, both settings yield the same positive long-run consumption level determined by the agent's participation constraint evaluated at current and future assets equal to zero. This is in contrast with the first best (see Proposition 1), where consumption converges to zero in the long run. The reason is that in the MPE and one-sided commitment insurance contracts the agent can always go to his outside option if offered consumption that is too low.

\section{Extensions}

\subsection{Non-contractible assets}

The results in Sections 2.3, 3.1, and 3.3 show that when the insurer lacks the ability to commit to a longterm contract, there are nontrivial equilibrium asset dynamics. Given that, in general, the agent holds assets 
in an MPE, a natural question is whether a Markov-perfect insurance contract would differ if the insurer were unable to control the agent's observable savings. This is important, as one can imagine many situations in which private or public insurance providers can observe or infer agents' asset positions but cannot force agents to hold particular asset levels or, equivalently, control agents' consumption directly. Below, we show that as long as the insurer obtains a positive share of the joint surplus, which contract side (insurer or agent) controls the savings choice does affect the nature of the MPE (see Section 4.2 for the remaining case of perfectly-competitive insurers).

Assume the agent's assets are observable but non-contractible. Thus, the insurer must induce the desired levels of $c$ and $a^{\prime}$ through a suitable choice of transfers. Consider the problem of an agent with current assets $a \in \mathbb{A}$ who signs an insurance contract that specifies transfers $\tau^{i}, i=1, \ldots, n$ :

$$
\max _{\left\{a^{i} \in \mathbb{A}\right\}_{i=1}^{n}} \sum_{i=1}^{n} \pi_{i}\left[u\left(r a+\tau^{i}-a^{i}\right)+\beta B\left(a^{i}\right)\right],
$$

where again we used Lemma 1 to obtain the agent's continuation value. The first-order condition with respect to savings, $a^{i}, i=1, \ldots, n$ is

$$
-u_{c}\left(c^{i}\right)+\beta B_{a}\left(a^{i}\right)+\xi^{i}=0,
$$

where $\xi^{i} \geq 0$ denotes the multiplier on the non-negativity constraint for $a^{i}$. We use the agent's first-order conditions in $a^{i}(15)$ as incentive-compatibility constraints for the insurer with respect to the agent's choice of $a^{i}$. This "first-order approach" is valid here since, by our assumptions on $u$ and $B$, the agent's problem is concave in $a^{i}$. The insurer's problem when the agent's assets are non-contractible is thus analogous to the problem with contractible assets from Section 3.1, (6)-(7), but with the following additional constraints:

$$
u_{c}\left(c^{i}\right)-\beta B_{a}\left(a^{i}\right) \geq 0 \text { for } i=1, \ldots, n .
$$

As in Section 3.1, it is easy to verify that the first-order conditions are symmetric across $i$ and so $c^{i}=c$ and $a^{i}=a^{\prime}$ for $i=1, \ldots, n$, again taking a symmetric solution. A Markov-perfect equilibrium with noncontractible assets, consisting of the functions $\left\{\mathcal{T}^{N}, \mathcal{A}^{N}, \Pi^{N}\right\}$, is then defined analogously to Definition 1 but with the additional incentive-compatibility constraints, (16) included in the insurer's problem. A Markovperfect contract with non-contractible assets for any given $a \in \mathbb{A}$ is simply the offered equilibrium transfer $\left\{\tau=\mathcal{T}^{N}(a)\right\}$.

Although the agent's savings $a^{\prime}$ are no longer contractible, his consumption and current assets are still observable. Therefore, as in Section 3.1 we can rewrite the problem of the insurer as if choosing consumption, $c$ (rather than transfers), and next-period asset $a^{\prime}$ subject to the participation constraint and the incentivecompatibility constraint for $a^{\prime}$,

$$
\Pi^{N}(a)=\max _{c, a^{\prime} \in \mathbb{A}} \bar{y}+r a-c-a^{\prime}+R^{-1} \Pi^{N}\left(a^{\prime}\right)
$$

subject to

$$
\begin{aligned}
u(c)+\beta B\left(a^{\prime}\right)-B(a) & =0 \\
u_{c}(c)-\beta B_{a}\left(a^{\prime}\right) & \geq 0, \text { with equality if } a^{\prime}>0 .
\end{aligned}
$$

Let $a^{\prime N}=\mathcal{A}^{N}(a)$ and $c^{N}=\mathcal{C}^{N}(a)$ be the optimal asset and consumption choices in the non-contractible assets problem (17)-(19). The following result characterizes some properties of Markov-perfect contracts with non-contractible assets.

Lemma 2. In an MPE with non-contractible assets: (i) consumption, $\mathcal{C}^{N}(a)$, is strictly increasing in a; (ii) savings, $\mathcal{A}^{N}(a)$, are strictly increasing in a at an interior solution and $\mathcal{A}^{N}(a)=0$ otherwise.

For the remainder of this section, we make the following assumption (see the discussion after Proposition 2 regarding $\hat{a})$.

Assumption 4. (i) $\bar{a}>\hat{a}$ and (ii) $\exists a \in(0, \min \{\hat{a}, \mathcal{A}(\bar{a})\}]$ such that $\frac{B_{a}(a)}{u_{c}\left(u^{-1}(B(a)-\beta B(0))\right)} \neq r$. 
An example that satisfies Assumption 4(ii) is $B(a)=\sqrt{r a+1}$ and $u(c)=\sqrt{c}$, while $B(a)=\sqrt{r a}$ and $u(c)=\sqrt{c}$ do not satisfy Assumption 4 (and also violate Assumption 2(ii)). An alternative sufficient condition to Assumption 4(ii) is to set $B(0)=0$ and take $u$ and $B$ satisfying the previous assumptions and such that $u^{-1}(B(a))$ is non-affine in $a$ or affine but with a slope different from $r$. The numerical example at the end of this subsection also satisfies Assumption 4.

The next Proposition characterizes the role of asset contractibility in Markov-perfect insurance contracts.

\section{Proposition 4. Asset contractibility in Markov-perfect contracts.}

(i) Under Assumptions 1, 2 and $4, M P E$ with contractible assets, $\{\mathcal{A}, \mathcal{C}, \Pi\}$ differ from $M P E$ with noncontractible assets, $\left\{\mathcal{A}^{N}, \mathcal{C}^{N}, \Pi^{N}\right\}$. That is, there exists at least one $a \in \mathbb{A}$ for which $\{\mathcal{A}(a), \mathcal{C}(a), \Pi(a)\} \neq$ $\left\{\mathcal{A}^{N}(a), \mathcal{C}^{N}(a), \Pi^{N}(a)\right\}$-it matters who controls asset accumulation.

(ii) Specifically, take any asset level $a \in(\hat{a}, \bar{a}]$ for which $a^{\prime}=\mathcal{A}(a)>0$ and $a^{\prime N}=\mathcal{A}^{N}(a)>0$. For any such $a$, if $\Pi_{a}\left(a^{\prime}\right)<0$, then savings are strictly higher and consumption lower in the non-contractible assets case: $a^{\prime}<a^{\prime N}$ and $c>c^{N}$. The opposite is true if $\Pi_{a}\left(a^{\prime}\right)>0$. It is not possible that $\Pi_{a}\left(a^{\prime}\right)=0$ for all $a \in(\hat{a}, \bar{a}]$.

Recall from Proposition 1 that the first-best contract remains incentive compatible when savings are noncontractible since the agent has no incentive to save given the full insurance provided and the fact that any side savings cannot alter the terms of the contract set at time zero. This is no longer the case here. The lack of commitment friction misaligns the asset accumulation incentives of the contracting parties. Intuitively, the agent can use his ability to save privately to increase his outside option, ensuring higher future transfers, which counters the principal's driving him to the lower bound $B(0)$.

Proposition 4 shows the existence of one or more asset levels for which the contracts with and without asset contractibility differ. It does not rule out the possibility that these two contracts coincide for some asset levels. Theoretically, this could occur-for example, if the MPE with non-contractible assets features a binding non-negativity constraint for $a$ close to zero.

To further characterize Markov-perfect insurance contracts with non-contractible assets solving (17)-(19), assume that $B(a)$ is twice continuously differentiable and focus on the case of an interior solution for assets, $a^{\prime}>0$, where $u_{c}(c)-\beta B_{a}\left(a^{\prime}\right)=0$. With $\lambda$ and $\mu$ the Lagrange multipliers associated with (18) and (19), respectively, the first-order conditions of problem (17)-(19) are

$$
\begin{aligned}
-1+\lambda u_{c}(c)+\mu u_{c c}(c) & =0 \\
-1+R^{-1} \Pi_{a}^{N}\left(a^{\prime}\right)+\lambda \beta B_{a}\left(a^{\prime}\right)-\mu \beta B_{a a}\left(a^{\prime}\right) & =0 .
\end{aligned}
$$

The system of equations formed by the constraints to the insurer's problem with non-contractible assets, (18)-(19) and its first-order conditions, (20)-(21), permits a steady-state with positive assets. In such a case, Markov-perfect contracts with and without asset contractibility differ for all $a \in A$ and imply different longrun allocations. Figure 2 shows a numerical example illustrating this point using the same parameterization as in Figure 1. As shown, long-run savings in the case of non-contractible assets are zero at some positive asset level. We also display profits, which are naturally lower for the non-contractible case. We omit displaying transfers and consumption since they are visually indistinguishable.

Figure 2: Effect of asset contractibility in MPE

[Figure 2 about here]

Note: Solid lines correspond to MPE with contractible assets; dashed lines correspond to MPE with non-contractible assets. The assumed parameterization is $u(c)=\ln c, \beta=0.93 ; r=1.06 ; R=1.07 ; y^{1}=0.1, y^{2}=0.3$, and $\pi^{1}=0.5$.

\subsection{Perfectly competitive insurers}

In this section, we characterize the case of a perfectly competitive insurance market with free entry by insurers, previously ruled out by Assumption 2(iv). Free entry implies that optimal contracts maximize the 
agent's expected present value utility subject to a zero-profits constraint for the insurer. More specifically, perfect competition in the insurance market results in zero per period profits for any submarket indexed by the agent's assets, $a$. Cross-subsidization across different asset levels or time periods is ruled out by the possibility of free entry each period.

Define a Markov-perfect equilibrium and Markov-perfect contracts analogously to Definition 1, basically replacing the agent's participation constraint with a zero-profit constraint for the insurer (details omitted to save space). Denoting $c^{i} \equiv r a+\tau^{i}-a^{i}$, the contracting problem of a perfectly competitive insurer under double-sided lack of commitment and when assets are contractible can be written recursively as ${ }^{14}$

$$
v(a)=\max _{\left\{\tau^{i}, a^{i} \in \mathbb{A}\right\}_{i=1}^{n}} \sum_{i=1}^{n} \pi_{i}\left[u\left(c^{i}\right)+\beta v\left(a^{i}\right)\right]
$$

subject to

$$
\sum_{i=1}^{n} \pi_{i}\left[y^{i}-\tau^{i}\right]=0 .
$$

There is no need for a participation constraint for the agent as he obtains all the surplus. It is easy to show, using the first-order conditions, that full insurance obtains once again, that is, $c^{i}=c, \tau^{i}=\tau$, and $a^{i}=a^{\prime}$ for all $i=1, \ldots, n$. Hence, from the zero-profits condition, $\tau=\bar{y}$ and $c=\bar{y}+r a-a^{\prime}$, which simplifies the above contracting problem to

$$
v(a)=\max _{a^{\prime} \in \mathbb{A}} u\left(\bar{y}+r a-a^{\prime}\right)+\beta v\left(a^{\prime}\right) .
$$

Under our assumptions on $u$ and $\beta r<1$, problem (22) is equivalent to Example 5.17 in SLP (pp. 126-28). Thus, the value function $v$ is continuous, strictly increasing, strictly concave, and continuously differentiable for any $a \in \mathbb{A}$. The policy function, $a^{\prime}=\mathcal{A}(a)$ is unique, continuous, and weakly increasing. Zero profits implies $\mathcal{T}(a)=\bar{y}$ for all $a \in \mathbb{A}$.

Proposition 5. In an MPE with free entry by insurers,

(i) at an interior solution for $a^{\prime}$, the standard Euler equation applies:

$$
u_{c}(c)=\beta r u_{c}\left(c^{\prime}\right)
$$

(ii) properties (i)-(iii) listed in Proposition 2 hold;

(iii) it does not matter whether agent's assets are contractible or not; that is, Markov-perfect contracts with contractible and non-contractible assets coincide for all $a \in \mathbb{A}$.

Contrast the Euler equation with free entry, (23), with its counterpart, (12), from the non-competitive case analyzed earlier. First, free entry and lack of commitment imply that no assets can be carried over time by the insurer - thus, only the agent's return $r$ matters. Second, the free-entry assumption implies that there is no conflict between the insurer and agent about the optimal time profile of consumption - it follows (23), which is precisely what the agent would do if he controlled his own savings. However, unless $r=R$, the parties' inability to commit still matters for the consumption path - the Euler equation in (23) does differ from that in the first best; see Proposition 1(iii). Third, note the contrast between the results of Proposition 5(iii) and Proposition 4 from the non-competitive case. Intuitively, a perfectly competitive insurer earning zero profits cannot manipulate the agent's future value by imposing a different savings path as an insurer with market power would.

The function $v(a)$ can be viewed as a limiting case of the outside option $B(a)$ used in previous sections, an upper bound for the agent's discounted utility for which the insurer will break even.

\footnotetext{
${ }^{14}$ See also Karaivanov and Martin (2012) for detailed discussion of the free-entry case in a more general insurance setting with moral hazard.
} 


\subsection{Bargaining}

So far, we have considered two extreme ways of dividing the surplus in insurance contracts. In Section 3 , we solved the problem of how much profit the principal can extract for a given agent outside option $B(a)$. Then, in Section 4.2, we solved the case of free entry by insurers in which all the surplus of an insurance contract goes to the agent. In this extension, we characterize Markov-perfect dynamic insurance when the insurer and the agent bargain over the contract terms every period. We adopt the proportional solution of Kalai (1977) and assume that the outside option for the agent is autarky $\Omega(a)$, as defined in Section 2, and the outside option for the principal is zero profits.

Let $\theta$ be the agent's bargaining weight, which in Kalai's proportional solution equals the agent's share of the total surplus. Focus on the case $\theta \in(0,1)$ so that the participation constraints of the contracting parties,

$$
\begin{aligned}
u(c)+\beta v^{K}\left(a^{\prime}\right)-\Omega(a) & \geq 0 \\
\bar{y}+r a-c-a^{\prime}+R^{-1} \Pi^{K}\left(a^{\prime}\right) & \geq 0,
\end{aligned}
$$

do not bind, where $\Pi^{K}$ denotes the insurer's profits and $v^{K}$ the agent's value function in this setting. As before, use $c \equiv r a+\tau-a^{\prime}$ to replace transfers with consumption and assets in all expressions.

The insurance contract with bargaining can be written as

$$
\max _{c, a^{\prime} \in \mathbb{A}} \bar{y}+r a-c-a^{\prime}+R^{-1} \Pi^{K}\left(a^{\prime}\right)
$$

subject to

$$
(1-\theta)\left[u(c)+\beta v^{K}\left(a^{\prime}\right)-\Omega(a)\right]-\theta\left[\bar{y}+r a-c-a^{\prime}+R^{-1} \Pi^{K}\left(a^{\prime}\right)\right]=0 .
$$

A Markov-perfect equilibrium with bargaining over the terms of the insurance contract is defined, in a manner similar to Definition 1, by a set of functions $\left\{\mathcal{C}^{K}, \mathcal{A}^{K}, v^{K}, \Pi^{K}\right\}$ that solve problem (24)-(25) for all $a \in \mathbb{A}$ and where

$$
\begin{aligned}
v^{K}(a) & =u\left(\mathcal{C}^{K}(a)\right)+\beta v^{K}\left(\mathcal{A}^{K}(a)\right) \\
\Pi^{K}(a) & =\bar{y}+r a-\mathcal{C}^{K}(a)-\mathcal{A}^{K}(a)+R^{-1} \Pi^{K}\left(\mathcal{A}^{K}(a)\right) .
\end{aligned}
$$

The resulting equilibrium value function $v^{K}(a)$ for the agent is an example of how to endogenize the agent's outside option function $B(a)$ from previous sections.

At an interior solution, the first-order conditions of problem (24)-(25) are

$$
\begin{aligned}
-1+\lambda\left[(1-\theta) u_{c}(c)+\theta\right] & =0 \\
-1+R^{-1} \Pi_{a}^{K}\left(a^{\prime}\right)+\lambda\left[(1-\theta) \beta v_{a}^{K}\left(a^{\prime}\right)-\theta\left(-1+R^{-1} \Pi_{a}^{K}\left(a^{\prime}\right)\right)\right] & =0,
\end{aligned}
$$

where $\lambda$ is the Lagrange multiplier associated with the surplus-splitting constraint, (25). From the first equation above, $\lambda=\left[(1-\theta) u_{c}(c)+\theta\right]^{-1}$ and, substituting in for $\lambda$ and simplifying, the second equation becomes

$$
-u_{c}(c)+\beta v_{a}^{K}\left(a^{\prime}\right)+R^{-1} u_{c}(c) \Pi_{a}^{K}\left(a^{\prime}\right)=0 .
$$

Problem (24)-(25) determines the insurer's equilibrium profits as a function of the agent's assets, $\Pi^{K}(a)$. By the Envelope Theorem, $\Pi_{a}^{K}(a)=r-\lambda\left[(1-\theta) \Omega_{a}(a)+\theta r\right]$, which, substituting in for $\lambda$ from above, implies

$$
\Pi_{a}^{K}(a)=\frac{r u_{c}(c)-\Omega_{a}(a)}{u_{c}(c)+\frac{\theta}{1-\theta}} .
$$

Using (26)-(27), the proportional surplus-splitting rule (25) in an MPE can be written as $(1-\theta)\left[v^{K}(a)-\right.$ $\Omega(a)]-\theta \Pi^{K}(a)=0$ for all $a \in \mathbb{A}$. Totally differentiating this expression with respect to $a$ and rearranging implies

$$
v_{a}^{K}(a)=\frac{\theta}{1-\theta} \Pi_{a}^{K}(a)+\Omega_{a}(a)
$$

Using (29), we obtain

$$
v_{a}^{K}(a)=\frac{u_{c}(c)\left[\frac{r \theta}{1-\theta}+\Omega_{a}(a)\right]}{u_{c}(c)+\frac{\theta}{1-\theta}} .
$$


Substituting in for (29) and (30) evaluated one period ahead into (28) and rearranging, we obtain the following Euler equation:

$$
u_{c}(c)=\beta R u_{c}\left(c^{\prime}\right)\left[\frac{\theta r+(1-\theta)\left[\Omega_{a}\left(a^{\prime}\right)-u_{c}(c)(R-r)(\beta R)^{-1}\right]}{\theta R+(1-\theta) \Omega_{a}\left(a^{\prime}\right)}\right] .
$$

Note that as the bargaining power of the agent $\theta \rightarrow 0$, equation (31) converges to

$$
u_{c}(c)=\beta R u_{c}\left(c^{\prime}\right)\left[1-\frac{(R-r)}{R} \frac{u_{c}(c)}{\beta \Omega_{a}\left(a^{\prime}\right)}\right] .
$$

If we set $B(a)=\Omega(a)$ in Section 3, then the Euler equation (12), which characterizes an interior solution of the Markov-perfect contracting problem with lack of commitment and a monopolistic insurer, coincides with the expression above. In other words, if the parties lack commitment and autarky is the agent's outside option, then as we decrease the agent's bargaining power toward zero in the bargaining problem (24)-(25), we converge to the Markov-perfect insurance contract with a monopolistic insurer. ${ }^{15}$

Alternatively, as the bargaining power of the agent $\theta \rightarrow 1$, expression (31) converges to $u_{c}(c)=\beta r u_{c}\left(c^{\prime}\right)$, which is the same as (23) - the Euler equation under free entry by insurers. In this case, as shown in Section 4.2 , the insurance contract does not depend on the agent's outside option.

\section{Concluding Remarks}

We study dynamic insurance problems in which the contracting parties cannot commit to long-term agreements and are able to carry resources over time at potentially different rates of return. We find that the gains from the insurer's commitment to an infinitely long contract, as opposed to one-period contracts, are derived from exploiting a superior rate of return on carrying assets over time. Perhaps surprisingly, there are no other efficiency gains from long-term commitment by the insurer in our setting. If the agent holds sufficiently low initial assets or if the insurer and agent share the same rate of return, one-period Markovperfect insurance contracts generate equivalent consumption paths and welfare as those arising when the insurer can commit to an infinitely long contract subject to per period participation constraints by the agent.

In contrast, an agent's ability to commit long term could be exploited by an insurer to generate higher ex-ante surplus by driving agent's consumption toward its lowest bound. This is impossible if the agent is free to leave the contract each period for an outside option with present value exceeding the value of zero consumption forever. Thus, lack of long-term commitment by the agent always introduces inefficiency relative to the first best if the optimal consumption profile is decreasing in time (the case $\beta R<1$ in our setting). The insurer's inability to commit and hence, the need to carry assets over time at the agent's inferior rate of return $(r)$, implies that assets accumulation and contractibility play a key role in Markov-perfect insurance contracts, yielding nontrivial asset dynamics. This is in contrast to the trivial role of observable assets in contracts with commitment.

The time profiles of the agent's consumption and the insurer's profits depend critically on the parties' ability to commit and the degree of the insurer's market power. If the insurer can commit to a long-term contract, production efficiency and total surplus are maximized since the agent's assets can be immediately invested in the superior return technology in exchange for promises of future consumption. In contrast, if insurers lack commitment and there is free entry, no assets can be carried at the superior rate of return in a Markov-perfect equilibrium - instead, the agent's initial assets are carried via the low-return savings technology until they are depleted. When the insurer has market power, the lack of commitment limits his ability to efficiently extract the agent's assets (they can be driven to zero only gradually), leading to distorted consumption and profit profiles and lower total surplus.

Our approach and findings can be related more generally to the literature on incomplete contracts and political economy. ${ }^{16}$ For example, Battaglini and Palfrey (2011) study a dynamic problem of surplus splitting

\footnotetext{
${ }^{15}$ More generally, if we solve the bargaining problem for an exogenously given agent's outside option $B(a)$ satisfying Assumption 2 , then we would also obtain (12) as $\theta \rightarrow 0$.

${ }^{16}$ We thank an anonymous referee for pointing out this connection.
} 
in a voting game. Each period, one of three agents proposes a sharing rule which is accepted if there is a majority in favor or, if rejected, the last-period's sharing rule is applied. Therefore, as in our analysis, a policy chosen today affects the next period's status quo and determines tomorrow's outside option. Battaglini and Palfrey also focus on Markov strategies and equilibria dependent only on payoff-relevant events. Experimentally, they find evidence for concave utility and show that agents' discount factors significantly affect the resulting equilibria.

In a companion paper (Karaivanov and Martin, 2012), we extend some of the results presented here to a setting in which income is endogenous and there is a moral hazard problem. We find that such an environment can account for a significant fraction of observed wealth inequality. We further show that at low asset levels, the welfare costs due to lack of commitment are larger than those due to moral hazard, while the opposite holds for high asset levels.

\section{References}

[1] Acemoglu, D., M. Golosov and A. Tsyvinski (2006), "Markets Versus Governments: Political Economy of Mechanisms", NBER Working Paper \#12224.

[2] Acemoglu, D., M. Golosov and A. Tsyvinski (2008), "Political Economy of Mechanisms", Econometrica $76(3): 619-641$.

[3] Aiyagari, S.R. (1994), "Uninsured Idiosyncratic Risk and Aggregate Saving", Quarterly Journal of Economics 109(3): 659-684.

[4] Allen, F. (1985), "Repeated principal-agent relationships with lending and borrowing", Economics Letters 17(1-2): 27-31.

[5] Battaglini, M. and T. R. Palfrey (2011), "The Dynamics of Distributive Politics", Economic Theory 49: 739-777.

[6] Bester, H. and R. Strausz (2001), "Contracting with Imperfect Commitment and the Revelation Principle: The Single Agent Case", Econometrica 69(4): 1077-1098.

[7] Bisin, A. and A. Rampini (2006), "Markets as beneficial constraints on the government", Journal of Public Economics 90(4-5): 601-629.

[8] Cole, H. and N. Kocherlakota (2001), "Efficient Allocations with Hidden Income and Hidden Storage", Review of Economic Studies 68(3): 523-542.

[9] Doepke, M. and R. Townsend (2006), "Dynamic mechanism design with hidden income and hidden actions", Journal of Economic Theory 126(1): 235-285.

[10] Fernandes, A. and C. Phelan (2000), "A Recursive Formulation for Repeated Agency with History Dependence", Journal of Economic Theory 91: 223-247.

[11] Kalai, E. (1977), "Proportional solutions to bargaining situations: Interpersonal utility comparisons", Econometrica 45: 1623-1630.

[12] Karaivanov, A. and F. Martin (2012), "Moral Hazard and Lack of Commitment in Dynamic Economies", manuscript, Simon Fraser University.

[13] Kocherlakota, N. (1996), "Implications of Efficient Risk Sharing without Commitment", Review of Economic Studies 63(4): 595-609.

[14] Kovrijnykh, N. (2012), "Debt Contracts with Partial Commitment", American Economic Review, forthcoming.

[15] Krueger, D. and H. Uhlig (2006), "Competitive risk-sharing contracts with one-sided commitment", Journal of Monetary Economics 53, pp. 1661-1691. 
[16] Liebhafsky, H., (1969), "New Thoughts about Inferior Goods", American Economic Review 59(5): 93134 .

[17] Ligon, E., J. Thomas and T. Worrall, (2002) "Informal Insurance Arrangements with Limited Commitment: Theory and Evidence from Village Economies", Review of Economic Studies 69(1): 209-244.

[18] Maskin, E. and J. Tirole (2001), "Markov Perfect Equilibrium", Journal of Economic Theory 100(2): $191-219$.

[19] Phelan, C. (1995), "Repeated Moral Hazard and One-Sided Commitment", Journal of Economic Theory 66(2): 488-506.

[20] Schechtman, J. and V. Escudero (1977), "Some Results on an Infinite Fluctuation Problem", Journal of Economic Theory 16: 151-166.

[21] Sleet, C. and S. Yeltekin (2006), "Credibility and Endogenous Societal Discounting", Review of Economic Dynamics 9: 410-437.

[22] Sleet, C. and S. Yeltekin (2008), "Politically Credible Social Insurance", Journal of Monetary Economics 55: $129-151$.

[23] Stokey, N., R. Lucas and E. Prescott (1989), Recursive Methods in Economic Dynamics, Harvard University Press, Cambridge, MA.

[24] Thomas, J. and T. Worrall (1988), "Self-Enforcing Wage Contracts", Review of Economic Studies 55(4): $541-553$.

[25] Thomas, J. and T. Worrall (1994), "Foreign Direct Investment and the Risk of Expropriation", Review of Economic Studies 61(1): 81-108. 


\section{Appendix A. Proofs}

\section{Appendix A.1. Proposition 1}

(i) Let $y_{t} \in\left\{y^{1}, \ldots, y^{n}\right\}$ be the realization of output in period $t \geq 0$ and let $s^{t} \equiv\left\{y_{0}, y_{1}, \ldots, y_{t}\right\}$ denote the history of output states up to $t$. Denote by $\eta\left(s^{t}\right)$ the probability of output history $s^{t}$ defined recursively as $\eta\left(s^{t}\right)=\eta\left(\left\{s^{t-1}, y^{i}\right\}\right)=\pi_{i} \eta\left(s^{t-1}\right)$, with $\eta\left(s^{-1}\right)=1$ for $i=1, \ldots, n$.

The first-best insurance contract $\left\{\tau^{*}\left(s^{t}\right), a^{*}\left(s^{t}\right)\right\}_{t=0}^{\infty}$ solves the problem,

$$
\max _{\left\{\tau\left(s^{t}\right), a\left(s^{t}\right) \geq 0\right\}} \sum_{t \geq 0, s^{t}} R^{-t} \eta\left(s^{t}\right)\left[y_{t}-\tau\left(s^{t}\right)\right]
$$

subject to the agent's ex-ante participation constraint

$$
\sum_{t \geq 0, s^{t}} \beta^{t} \eta\left(s^{t}\right) u\left(c\left(s^{t}\right)\right)-B\left(a_{0}\right) \geq 0,
$$

where $c\left(s^{t}\right) \equiv \operatorname{ra}\left(s^{t-1}\right)+\tau\left(s^{t}\right)-a\left(s^{t}\right)$ is agent's consumption and $a\left(s^{-1}\right)=a_{0} \in \mathbb{A}$ are the agent's initial assets. We can rewrite the above problem in a mathematically equivalent way in terms of $\left\{c\left(s^{t}\right), a\left(s^{t}\right)\right\}_{t=0}^{\infty}$ as:

$$
\max _{\left\{c\left(s^{t}\right), a\left(s^{t}\right) \geq 0\right\}} \sum_{t \geq 0, s^{t}} R^{-t} \eta\left(s^{t}\right)\left[y_{t}-c\left(s^{t}\right)\right]-\sum_{t \geq 0, s^{t}} R^{-t-1} \eta\left(s^{t}\right) a\left(s^{t}\right)[R-r]+r a_{0}
$$

subject to (A.2). Since $r \leq R$ and given $a\left(s^{t}\right) \geq 0$, it is clearly optimal to set $a^{*}\left(s^{t}\right)=0$ for any $s^{t}, t \geq 0$. Intuitively, "extracting" agent's assets at time zero is optimal in the first best (strictly if $r<R$ ) since allowing the agent to carry assets over time at the inferior return $r$ destroys surplus.

(ii)-(iii) To simplify the notation, call $a_{t+1}^{i} \equiv a\left(s^{t}\right)$ and $c_{t}^{i} \equiv c\left(s^{t}\right)$ when $y_{t}=y^{i}$, for $i=1, \ldots, n$. The first-order condition of problem (A.3) with respect to $c_{t}^{i}$ implies

$$
-R^{-t}+\beta^{t} \lambda u_{c}\left(c_{t}^{i}\right)=0,
$$

where $\lambda$ is the multiplier on the participation constraint. Equation (A.4) implies full insurance: $c\left(s^{t-1}, y^{i}\right)=$ $c_{t}^{*}$ for all $t, s^{t-1}, i=1, \ldots, n$-part (ii). Given this, we have (taking ratios in (A.4)),

$$
u_{c}\left(c_{t}\right)=\beta R u_{c}\left(c_{t+1}\right)
$$

which yields part (iii).

(iv) Suppose, at the first-best contract, the agent wanted to privately save an extra unit of assets. His first-order optimality condition for choosing $a_{t+1} \geq 0$ is $u_{c}\left(c_{t}\right) \geq \beta r u_{c}\left(c_{t+1}\right)$ with equality when $a_{t+1}>0$. The agent's cost of saving an extra unit in today's utility is thus $u_{c}\left(c_{t}^{*}\right)$ which equals $\beta R u_{c}\left(c_{t+1}^{*}\right)$ by (A.5), while his gain is $\beta r u_{c}\left(c_{t+1}^{*}\right)$. Since $R \geq r$, the cost is weakly larger than the gain. Thus, the agent would not wish to save privately if given the first-best consumption sequence $\left\{c_{t}^{*}\right\}_{t=0}^{\infty}$

\section{Appendix A.2. Lemma 1}

Consider problem (4) subject to (5). For all $a \in \mathbb{A}$, let $\mathcal{T}(a)$ and $\mathcal{A}(a)$ be the optimal transfer and asset choices, which imply optimal consumption $\mathcal{C}(a) \equiv r a+\mathcal{T}(a)-\mathcal{A}(a)$. The agent's continuation utility is $v(a) \equiv u(\mathcal{C}(a))+\beta v(\mathcal{A}(a))$. Participation by the agent implies $v(a) \geq B(a)$ for all $a \in \mathbb{A}$. We have to show that the participation constraint (5) is satisfied with equality for all $a$, i.e., that $u(\mathcal{C}(a))+\beta v(\mathcal{A}(a))=B(a)$, which would imply $v(a)=B(a)$ for all $a$.

By Assumption 2, there exist $c>0$ and $a^{\prime} \in \mathbb{A}$ such that $u(c)+\beta B\left(a^{\prime}\right)=B(a)$ for all $a \in \mathbb{A} .{ }^{17}$ Take any $\tilde{a} \in \mathbb{A}$ and suppose optimal consumption is positive. Then, the agent's participation constraint must be

\footnotetext{
${ }^{17}$ By Assumption 2(ii), there exists $\hat{c}>0$ such that $u(\hat{c})+\beta B(0)=B(0)$; thus, at $a=0$, there is a combination of consumption and future assets that satisfies the participation constraint. Assumption 2(iii), plus the fact that $u$ and $B$ are strictly increasing also ensures that there exists a $\bar{c}>0$ such that $u(\bar{c})+\beta B(0)=B(\bar{a})$. Hence, by continuity, there always exists positive consumption and admissible asset choice that satisfy the participation constraint with equality for all $a \in \mathbb{A}$.
} 
satisfied with equality and so $v(\tilde{a})=B(\tilde{a})$; otherwise, the insurer can reduce agent's current consumption (reduce $\mathcal{T}(\tilde{a})$ ) and earn higher profits.

Next, suppose that optimal consumption for $\tilde{a}$ is zero, the optimal asset choice is positive and the participation constraint holds with strict inequality, i.e., $u(0)+\beta v(\mathcal{A}(\tilde{a}))>B(\tilde{a}) .{ }^{18}$ Consider the continuation of this contract. By Assumption 2, consumption cannot remain zero forever; otherwise we will have $v(\tilde{a})=$ $\frac{u(0)}{1-\beta}<B(0) \leq B(\tilde{a})$ - a contradiction. Thus, there exists a future period with associated assets $\tilde{\tilde{a}}$, for which consumption in the following period switches to positive for the first time and thus, by the earlier argument, we have $v(\mathcal{A}(\tilde{\tilde{a}}))=B(\mathcal{A}(\tilde{\tilde{a}}))$. Suppose $u(0)+\beta B(\mathcal{A}(\tilde{\tilde{a}}))>B(\tilde{\tilde{a}})$ and $\mathcal{A}(\tilde{\tilde{a}})>0$ is optimal (see below for the case when both future assets and consumption are zero). Given that $B(\mathcal{A}(\tilde{\tilde{a}}))$ is equal to the discounted sum of future utility of consumption by the agent, the insurer at $\tilde{\tilde{a}}$ can reduce $\mathcal{A}(\tilde{\tilde{a}})$ slightly, which decreases the continuation value for the agent while still satisfying participation. Thus, the discounted sum of future utility of consumption decreases, which implies a lower discounted sum of future transfers and hence, higher profits for the insurer-a contradiction. Suppose instead $u(0)+\beta B(\mathcal{A}(\tilde{\tilde{a}}))=B(\tilde{\tilde{a}})$. This implies $v(\tilde{\tilde{a}})=B(\tilde{\tilde{a}})$. Go back in time towards the period with assets $\tilde{a}$ where we started. Since we assumed $u(0)+\beta v(\mathcal{A}(\tilde{a}))>B(\tilde{a})$, at some point in this "going back" process we must have $u(0)+\beta v(\mathcal{A}(a))>B(a)$. Again, if $\mathcal{A}(a)>0$ then there is a profitable deviation as shown above.

To conclude the proof, we also need to rule out the case of zero consumption and zero future assets at optimum. If $\mathcal{C}(\tilde{a})=\mathcal{A}(\tilde{a})=0$, then

$$
v(\tilde{a})=u(0)+\beta v(0) \geq B(\tilde{a})>\frac{u(0)}{1-\beta},
$$

where the last inequality follows from Assumption 2. If $\tilde{a}=0$ then (A.6) implies $v(0)=\frac{u(0)}{1-\beta} \geq B(0)>\frac{u(0)}{1-\beta}$, a contradiction. Thus, $\mathcal{C}(0)>0$ and/or $\mathcal{A}(0)>0$. As shown above, in any of these cases the participation constraint binds; thus, we obtain $v(0)=B(0)$. Now suppose $\mathcal{C}(\tilde{a})=\mathcal{A}(\tilde{a})=0$ for some $\tilde{a}>0$. Then $v(\tilde{a})=u(0)+\beta v(0) \geq B(\tilde{a})$. Given $v(0)=B(0)$, we have $u(0)+\beta B(0) \geq B(\tilde{a})$ and therefore, $u(0) \geq$ $B(\tilde{a})-\beta B(0)>(1-\beta) B(0)$, which contradicts Assumption 2(ii). Thus, this case is impossible as well and, overall, we obtain $v(a)=B(a)$ for all $a \in \mathbb{A}$.

\section{Appendix A.3. Proposition 2}

(i) and (ii) To show the properties of assets and consumption in an MPE we first characterize the properties of a solution when the non-negativity constraint on asset choice binds. Next, we characterize the properties of an interior solution. Finally, we characterize the critical asset level $\hat{a}$. Note that we will show below that the constraint $a^{\prime} \leq \bar{a}$ never binds, as implied by the result $\mathcal{A}(a)<a$ for all asset levels with an interior solution.

\section{Appendix A.3.1. Corner solution in assets}

Suppose $a^{\prime} \geq 0$ binds at some $a \in \mathbb{A}$. From (7) we have $u(\mathcal{C}(a))+\beta B(0)=B(a)$. Since $u$ and $B$ are strictly increasing, this implies that consumption $\mathcal{C}(a)$ is strictly increasing for any such $a$. In the following period, we have $u(\mathcal{C}(0))+\beta B(\mathcal{A}(0))=B(0)$. If $a>0$, then $u(\mathcal{C}(a))+\beta B(0)=B(a)>B(0)=u(\mathcal{C}(0))+\beta B(\mathcal{A}(0))$ and so, $u(\mathcal{C}(a))>u(\mathcal{C}(0))+\beta(B(\mathcal{A}(0))-B(0))$. Given that $\mathcal{A}(0) \in \mathbb{A}$ is non-negative and $B$ is strictly increasing, this implies $u(\mathcal{C}(a))>u(\mathcal{C}(0))$ and hence, $c=\mathcal{C}(a)>\mathcal{C}(0)=c^{\prime}$, which implies a decreasing consumption time-profile for any such $a$ (strictly if $a>0$ ).

\section{Appendix A.3.2. Interior solution in assets}

Now suppose $a^{\prime} \geq 0$ does not bind at $a \in \mathbb{A}$. At an interior solution, (10) implies

$$
R-r=B_{a}\left(a^{\prime}\right)\left[\frac{\beta R}{u_{c}(c)}-\frac{1}{u_{c}\left(c^{\prime}\right)}\right] .
$$

Given Assumption 1 ( $R \geq r$ and $\beta R \leq 1$ but both do not hold at equality simultaneously) and Assumption 2(i) ( $B(a)$ strictly increasing), the above equation implies $u_{c}(c)<u_{c}\left(c^{\prime}\right)$. Thus, we obtain $c>c^{\prime}$, i.e., once again, a decreasing consumption time-profile.

\footnotetext{
${ }^{18}$ Note that if we assume $u(0)=-\infty$ this case is impossible.
} 
Next we show that $\mathcal{A}(a)<a$. Suppose not. Then $\mathcal{A}(a)=a^{\prime} \geq a$ for some $a \in \mathbb{A}$ and so, using (7), $u(c)+\beta B\left(a^{\prime}\right)=B(a) \leq B\left(a^{\prime}\right)=u\left(c^{\prime}\right)+\beta B\left(a^{\prime \prime}\right)$. Since $c^{\prime}<c$, as shown above, this implies $a^{\prime \prime}>a^{\prime}$. Given that, from Appendix A.3.1 and the previous paragraph, the consumption time-profile is strictly decreasing for all $a \in(0, \bar{a}]$, we can apply the same argument repeatedly and thus, assets strictly increase over time. There are two possibilities: either assets hit the upper bound $\bar{a}$ or they converge to some $a^{*} \in(0, \bar{a}]$. With multiplier $\mu \geq 0$ associated with the constraint $a^{\prime} \leq \bar{a}$, the first-order condition (A.7) becomes

$$
R-r+R \mu=B_{a}\left(a^{\prime}\right)\left[\frac{\beta R}{u_{c}(c)}-\frac{1}{u_{c}\left(c^{\prime}\right)}\right],
$$

which again implies $c>c^{\prime}$. Thus, for any $a$ with $a^{\prime}=\bar{a}$, we have $u(c)+\beta B(\bar{a})=B(a) \leq B(\bar{a})=$ $u\left(c^{\prime}\right)+\beta B\left(a^{\prime \prime}\right)$. Given $c>c^{\prime}$ it must be that $a^{\prime \prime}>\bar{a}$, a contradiction with $a^{\prime \prime} \in \mathbb{A}$. Now consider the case of assets converging to $a^{*} \in(0, \bar{a}]$. In the limit, $u\left(c^{*}\right)=(1-\beta) B\left(a^{*}\right)>(1-\beta) B(a)$, since as argued above, assets strictly increase over time. But then, given our assumption that $a^{\prime} \geq a$, the participation constraint $u(c)+\beta B\left(a^{\prime}\right)=B(a)$ implies $u(c)=B(a)-\beta B\left(a^{\prime}\right) \leq(1-\beta) B(a)$. Thus, $u(c)<u\left(c^{*}\right)$, which contradicts a strictly decreasing consumption time-profile. It follows that $a^{\prime}<a$ at an interior solution.

We now show that consumption, $\mathcal{C}(a)$ is strictly increasing in assets $a$. Suppose not. Then the participation constraint $u(\mathcal{C}(a))+\beta B(\mathcal{A}(a))=B(a)$ implies that $\mathcal{A}(a)$ is strictly increasing (since $B(a)$ is strictly increasing). We also showed that $c>c^{\prime}$, i.e., $\mathcal{C}(a)>\mathcal{C}(\mathcal{A}(a))$ which, if $\mathcal{C}$ is decreasing, implies $a \leq \mathcal{A}(a)$, a contradiction with the strictly decreasing asset time-profile shown above.

We finally show that $a^{\prime}=\mathcal{A}(a)$ is strictly increasing in $a$ for the case of interior solution. Suppose not. Then, since $\mathcal{C}(a)$ is strictly increasing and $u$ and $B$ are strictly concave, we obtain a contradiction since the right-hand side of (A.7) is strictly increasing in $a$, while the left-hand side is constant in $a$. To see this, note that given $\mathcal{C}(a)$ is strictly increasing, if we assume that $\mathcal{A}(a)$ is non-increasing, the terms $B_{a}\left(a^{\prime}\right)$ and $-\frac{1}{u_{c}\left(c^{\prime}\right)}$ are increasing in $a$ and the term $\frac{\beta R}{u_{c}(c)}$ is strictly increasing in $a$. Thus, $\mathcal{A}(a)$ is strictly increasing at an interior solution.

\section{Appendix A.3.3. The critical point $\hat{a}$}

We first show that $a^{\prime} \geq 0$ binds at $a=0$. Suppose not, i.e., the solution is interior. Then, as shown above, we must have $a^{\prime}<a=0$, a contradiction. Assumptions 1 and 2 imply $R-r>\frac{B_{a}(0)(\beta R-1)}{u_{c}(\mathcal{C}(0))}$ since the left-hand side is non-negative, the right-hand side is non-positive, and $R=r$ and $\beta R=1$ cannot hold simultaneously. Note that $\frac{B_{a}(0)(\beta R-1)}{u_{c}(\mathcal{C}(0))}$ is the right hand side of (A.7) evaluated at $a=a^{\prime}=0$. Take $a=\varepsilon$, with $\varepsilon$ sufficiently close to zero. This implies that the following inequality must hold:

$$
R-r>B_{a}(\mathcal{A}(\varepsilon))\left[\frac{\beta R}{u_{c}(\mathcal{C}(\varepsilon))}-\frac{1}{u_{c}(\mathcal{C}(\mathcal{A}(\varepsilon)))}\right] .
$$

For $\mathcal{A}(\varepsilon) \geq 0$, (A.8) holds by the continuity of $u_{c}$ and $\mathcal{C}(a)$, the strict concavity of $B$ and our previous result that $\mathcal{C}(a)$ is strictly increasing. Thus, (10) implies that $a^{\prime} \geq 0$ binds, i.e., $\mathcal{A}(\varepsilon)=0$.

Consider the right-hand side of (A.8) when $a^{\prime}=\mathcal{A}(\varepsilon)=0$, namely $B_{a}(0)\left[\frac{\beta R}{u_{c}(\mathcal{C}(a))}-\frac{1}{u_{c}(\mathcal{C}(0))}\right]$. It is strictly increasing in $a$ by our assumptions on $u$ and $B$ and the result that $\mathcal{C}(a)$ is strictly increasing. There are two possibilities. Either $R-r>B_{a}(0)\left[\frac{\beta R}{u_{c}(\mathcal{C}(\bar{a}))}-\frac{1}{u_{c}(\mathcal{C}(0))}\right]$, in which case by (10) we have $a^{\prime}=0$ for all $a \in \mathbb{A}$, or $\exists \alpha \in(0, \bar{a}]$ such that $R-r=B_{a}(0)\left[\frac{\beta R}{u_{c}(\mathcal{C}(\alpha))}-\frac{1}{u_{c}(\mathcal{C}(0))}\right]$ in which case $a^{\prime}=0$ for all $a \in[0, \alpha]$ (a corner solution) and $a^{\prime}>0$ for all $a \in(\alpha, \bar{a}]$ (an interior solution). Let $\hat{a}=\bar{a}$ in the former case and $\hat{a}=\alpha$ in the latter.

(iii) We will show that for any $a_{0} \in \mathbb{A}$ the sequence $\left\{a_{k}\right\}_{k=0}^{\infty}$ with elements $a_{k} \equiv \mathcal{A}\left(a_{k-1}\right)$ for all $k \geq 1$ defining the time-profile of agent's assets converges to zero in finite time. Suppose first $a_{0} \in[0, \hat{a}]$. Then, by part (i) we have $a_{1}=a_{2}=\ldots=0$ and hence agent's assets converge to zero in one step.

Let now $a_{0}>\hat{a}$ (naturally, this is possible only if $\hat{a} \in(0, \bar{a})$ ). The sequence $\left\{a_{k}\right\}_{k=0}^{\infty}$ defined above is monotonically decreasing since $a_{k}=\mathcal{A}\left(a_{k-1}\right) \leq a_{k-1}$ by part (i) and bounded (all its elements are nonnegative). Thus, it is convergent. Call its limit $\tilde{a}$, i.e., $\mathcal{A}(\tilde{a})=\tilde{a}$. If $\tilde{a}>\hat{a}$ we obtain a contradiction since it must be that $\mathcal{A}(\tilde{a})<\tilde{a}$ by part (i). So it must be that $\tilde{a} \in[0, \hat{a}]$. But then $\mathcal{A}(\tilde{a})=0$ by part (i), so it must be that $\tilde{a}=0$. Thus far, we have shown that agent's assets converge to zero. 
We next show that the convergence to zero assets is in finite time. The definition of convergent sequence applied to $\left\{a_{k}\right\}$ and its limit $\tilde{a}=0$ implies that, $\forall \varepsilon>0$, there exists a natural number $N$ such that for all $n \geq N$ we have $\left|a_{n}-\tilde{a}\right|=a_{n}<\varepsilon$. Take some $\varepsilon \in(0, \hat{a})$. Then, there exists a finite $N_{\varepsilon}$ such that $a_{n}<\varepsilon<\hat{a}$ for any $n \geq N_{\varepsilon}$ - that is, $\mathcal{A}\left(a_{n}\right)=0$ for any such $n$. Therefore, assets converge to zero in at most $N_{\varepsilon}+1$ steps since $a_{N_{\varepsilon}+1}=\mathcal{A}\left(a_{N \varepsilon}\right)=0$.

Finally, we show that consumption is positive in the long-run. Using (7), long-run consumption $\mathcal{C}(0)$ solves $u(\mathcal{C}(0))=(1-\beta) B(0)$ and thus, by Assumption $2(\mathrm{ii}), \mathcal{C}(0)>0$.

\section{Appendix A.4. Proposition 3}

(i) Let $r=R$. Define $\hat{\Pi}(a) \equiv \Pi(a)-R a$ and rewrite the Markov-perfect contracting problem (6)-(7) as:

$$
\hat{\Pi}(a)=\max _{c, a^{\prime} \in \mathbb{A}} \bar{y}-c+\frac{\hat{\Pi}\left(a^{\prime}\right)}{R}
$$

subject to

$$
u(c)+\beta B\left(a^{\prime}\right)-B(a)=0 .
$$

Take the second-stage problem under one-sided commitment, (14) and do a change of variables from $w$ to $a$ by calling $a=B^{-1}(w)$ for any $w \in W$-by our assumptions $B$ is strictly monotonically increasing, hence invertible, and by the definition of $W=[B(0), B(\bar{a})]$, any $w \in W$ is in the range of $B(a)$ for $a \in \mathbb{A}$, i.e., $a=B^{-1}(w) \in \mathbb{A}$. Call $\bar{\Pi}(a) \equiv \tilde{\Pi}^{O S}(B(a))=\tilde{\Pi}^{O S}(w)$. With this change of variables, problem (14) for state $w(=B(a))$ is mathematically equivalent to the MPE problem (A.9) for state $a .{ }^{19}$ That is, any solution to the former problem is solution to the latter and vice versa. Consequently, $\bar{\Pi}(a)=\hat{\Pi}(a)$. From Proposition 2 , since $a^{\prime} \leq a$ for all $a \in \mathbb{A}$ in an MPE (strictly if $a>\hat{a}$ ), this equivalence implies that $w^{\prime} \leq w=B(a)$ for all $w \in W$ in (14) and so we verify that the upper bound of $W$ is not restrictive in the second-stage problem.

Look now at the first-stage of the one-sided commitment problem, (13) starting from some initial assets $a_{0} \in \mathbb{A}$. Changing variables from $w$ to $a^{\prime}$ by calling $a^{\prime}=B^{-1}(w)$ as before (again, this is possible for any $w \in W$ since $B$ is strictly increasing) and plugging in for $\tilde{\Pi}^{O S}(w)=\bar{\Pi}\left(a^{\prime}\right)=\hat{\Pi}\left(a^{\prime}\right)$, the first-stage problem, (13) is mathematically equivalent to:

$$
\max _{c_{0}, a^{\prime} \in \mathbb{A}} \bar{y}-c_{0}+\frac{\hat{\Pi}\left(a^{\prime}\right)}{R}+r a_{0}
$$

subject to

$$
u\left(c_{0}\right)+\beta B\left(a^{\prime}\right)-B\left(a_{0}\right)=0 .
$$

Again, since $B$ is strictly increasing, the constraint $a^{\prime} \in \mathbb{A}$ is equivalent to $B\left(a^{\prime}\right) \in[B(0), B(\bar{a})]$ which, with the change of variables, stands in for $w \in W$ in the first-stage problem. Clearly, problem (A.10) has the same solution $c_{0}, a^{\prime}$ as the Markov-perfect problem (A.9) starting from the same $a_{0}$.

In sum, we showed that the one-sided commitment and Markov-perfect problems initialized at the same asset level $a_{0}$ are mathematically equivalent to each other. Hence, the consumption and present-value profits paths (after adding back the $R a_{0}$ term to $\left.\hat{\Pi}\left(a_{0}\right)\right)$ generated by them coincide. This equivalence also implies a one-to-one mapping on the equilibrium path between promised utility in the one-sided commitment contract and asset levels in a Markov-perfect contract. By Proposition 2, since assets in an MPE decrease over time to zero (i.e., $w=B(a)$ decreases to $B(0))$ we again see that the upper bound on $W$ is not restrictive for any $a_{0} \in \mathbb{A}$ and that we can set $\bar{w}\left(a_{0}\right)=B\left(a_{0}\right)$.

In part (ii) below, we complete the proof of the proposition statement by showing equivalence of the two problems for the case $a_{0} \in[0, \hat{a}]$ with $r<R$.

(ii) Suppose now $r<R$. Define $\Pi^{*}(a) \equiv \Pi(a)-R a$ and re-write the MPE problem (6)-(7) as:

$$
\Pi^{*}(a)=\max _{c, a^{\prime} \in \mathbb{A}} \bar{y}-(R-r) a-c+\frac{\Pi^{*}\left(a^{\prime}\right)}{R}
$$

\footnotetext{
${ }^{19}$ Note that the constraint $w^{\prime} \in W$ in problem (14) can be rewritten as $B\left(a^{\prime}\right) \in[B(0), B(\bar{a})]$ and, since $B$ is strictly increasing, this is equivalent to $a^{\prime}=B^{-1}\left(w^{\prime}\right) \in \mathbb{A}$.
} 
subject to

$$
u(c)+\beta B\left(a^{\prime}\right)-B(a)=0 .
$$

Using analogous arguments as in part (i), the second-stage one-sided commitment problem (14) does not depend on $r$ and so remains the same as in part (i), i.e., mathematically equivalent to problem (A.9). Hence, when $r<R$ and $a>0$, problem (A.11) is no longer equivalent to problem (A.9), which is mathematically equivalent to the second-stage one-sided commitment problem, because of the extra term $(R-r) a$ in the objective.

In the special case $a_{0} \in[0, \hat{a}]$ we know from Proposition 2 that $a^{\prime}=0$ and hence agent's assets stay at zero forever after the initial period. Thus, in the second-stage problem (14) with assets $a=0$, the extra term $(R-r) a$ in (A.11) drops out restoring the consumption and profits paths equivalence between one-sided commitment and the Markov-perfect insurance as shown in part (i).

To obtain a more precise characterization, let $\left\{c_{t}^{M}, a_{t+1}^{M}\right\}_{t=0}^{\infty}$ be the consumption and assets sequences solving problem (A.11) starting from assets $a_{0}$ following the MPE policies $\mathcal{C}$ and $\mathcal{A}$. By repeatedly plugging in, we can write the associated present discounted value of profits at $t=0$ as:

$$
\Pi^{*}\left(a_{0}\right)=\sum_{t=0}^{\infty} R^{-t}\left[\bar{y}-c_{t}^{M}-(R-r) a_{t}^{M}\right]
$$

where $a_{0}^{M}=a_{0}$.

Using our results from part (i) regarding problems (A.9) and (A.10), the time-zero value of the onesided commitment problem's objective function evaluated at our MPE solution $\left\{c_{t}^{M}, a_{t+1}^{M}\right\}_{t=0}^{\infty}$ from $a_{0}$ is $r a_{0}+\sum_{t=0}^{\infty} R^{-t}\left[\bar{y}-c_{t}^{M}\right]$. Since the Markov-perfect (A.9) and one-sided commitment problems share the same set of constraints, the sequence $\left\{c_{t}^{M}, a_{t+1}^{M}\right\}_{t=0}^{\infty}$ is feasible in the one-sided problem from $a_{0}$ but not necessarily optimal. Thus, letting $\bar{\Pi}\left(a_{0}\right)$ be the maximized value in the one-sided commitment contract, we have:

$$
\bar{\Pi}\left(a_{0}\right) \geq r a_{0}+\sum_{t=0}^{\infty} R^{-t}\left[\bar{y}-c_{t}^{M}\right] \geq R a_{0}+\sum_{t=0}^{\infty} R^{-t}\left[\bar{y}-c_{t}^{M}-(R-r) a_{t}^{M}\right]=R a_{0}+\Pi^{*}\left(a_{0}\right)=\Pi\left(a_{0}\right) .
$$

The second inequality follows since $a_{0}^{M}=a_{0}$ and so $R a_{0}-\sum_{t=0}^{\infty} R^{-t}(R-r) a_{t}^{M}=r a_{0}-\sum_{t=1}^{\infty} R^{-t}(R-r) a_{t}^{M} \leq$ $r a_{0}$. Thus, since $R>r$, the second inequality is strict if at least one $a_{t}^{M}$ with $t \geq 1$ is strictly positive. By Proposition 2, this implies that, for any $a_{0}>\hat{a}$, present-value profits in MPE, $\Pi\left(a_{0}\right)$ are strictly lower than present-value profits with one-sided commitment, $\bar{\Pi}\left(a_{0}\right)$ since for any $a_{0}^{M}=a_{0}>\hat{a}$ we know that at least $a_{1}^{M}>0$ (and possibly other $a_{t}^{M}$ with $t>1$ are also positive).

For part ii(b) use the first-order conditions of the one-sided commitment problem, (13) and (14) to obtain $u_{c}(c)=\beta R u_{c}\left(c^{\prime}\right)$ at an interior solution. This coincides with the Euler equation in the first best, (A.5). Therefore, the discussion from Section 3.2 below equation (12) applies. When $\beta R<1$, the Euler equations, (12) and $u_{c}(c)=\beta R u_{c}\left(c^{\prime}\right)$, respectively for the Markov-perfect and one-sided commitment settings, imply that consumption is decreasing over time in both cases. From the agent's participation constraint, since $a=0$ in the long-run, long-run consumption is the same in both settings - the value $c^{*}>0$ solving $u\left(c^{*}\right)=$ $(1-\beta) B(0)$.

\section{Appendix A.5. Lemma 2}

For any $a \in \mathbb{A}$ such that $a^{\prime N}>0$ (interior solution), (19) and the strict concavity of $u$ and $B$ imply that $c^{N}$ and $a^{\prime N}$ must move in the same direction as $a$ varies. Therefore, since $u$ and $B$ are strictly increasing, (18) implies that $c^{N}$ and $a^{\prime N}$ are both strictly increasing in $a$. For any $a \in \mathbb{A}$ for which we do not have interior solution, constraint (19) does not bind and hence our previous arguments from Proposition 2 apply.

\section{Appendix A.6. Proposition 4}

(i) Suppose Markov-perfect contracts with contractible and non-contractible assets coincide for all $a \in \mathbb{A}$. Then, the contractible-assets contracts $\left\{c=\mathcal{C}(a), a^{\prime}=\mathcal{A}(a)\right\}$ solving problem (6)-(7) must satisfy constraint (19) in the non-contractible assets problem for any $a$. Consider the first-order condition with respect to $a^{\prime}$ in the contractible-assets problem: $\Pi_{a}\left(a^{\prime}\right) / R+\lambda\left[-u_{c}(c)+\beta B_{a}\left(a^{\prime}\right)\right]+\zeta=0$, and take some $a \in(\hat{a}, \bar{a}]$, 
i.e., with $\zeta=0$ and $a^{\prime}>0$. If $\Pi_{a}\left(a^{\prime}\right)<0$ for that $a$ then, since $\lambda=1 / u_{c}>0$ from the consumption first-order condition, we have $u_{c}(c)-\beta B_{a}\left(a^{\prime}\right)<0$ which violates (19). Conversely, if $\Pi_{a}\left(a^{\prime}\right)>0$ for that $a$, then $u_{c}(c)-\beta B_{a}\left(a^{\prime}\right)>0$ and so (19) implies $a^{\prime N}=0$, which differs from $a^{\prime}>0$. Thus, in both these cases the MPE contracts with and without asset contractibility differ at $a$. The only remaining possibility is $\Pi_{a}\left(a^{\prime}\right)=0$ for all $a \in(\hat{a}, \bar{a}]$ which, by Proposition $2(\mathrm{i})$, is equivalent to $\Pi_{a}(a)=0$ for all $a \in(0, \mathcal{A}(\bar{a})]$. We will show that this is ruled out by Assumption 4(ii). An alternative sufficient condition is discussed in the main text, below the Assumption statement.

Suppose $\Pi_{a}(a)=0$ for all $a \in(0, \mathcal{A}(\bar{a})]$. Take some $a \in A^{1} \equiv(0, \min \{\hat{a}, \mathcal{A}(\bar{a})\}]$ noting that $A^{1}$ is a subset of $(0, \mathcal{A}(\bar{a})]$. By Proposition 2 we know that $a^{\prime}=\mathcal{A}(a)=0$ for all $a \in A^{1}$. From the envelope condition, (9) we have $\Pi_{a}(a)=r-B_{a}(a) / u_{c}(\mathcal{C}(a))$. Using the agent's participation constraint $(7)$ to substitute for $\mathcal{C}(a)$, we obtain

$$
\Pi_{a}(a)=r-\frac{B_{a}(a)}{u_{c}\left(u^{-1}(B(a)-\beta B(0))\right)}
$$

which is different from zero by Assumption 4 for some $\tilde{a} \in A^{1}$. Hence, since $\mathcal{A}(a)$ is continuous and strictly increasing when positive (see Proposition 2), $\exists a>\hat{a}$ with $\tilde{a}=\mathcal{A}(a) \in A^{1}$ for which $\Pi_{a}(\tilde{a})$ is either $>0$ or $<0$ and so, as argued above, the non-contractible and contractible assets contracts differ at any such $a$.

(ii) The first-order condition of the contractible assets problem, (6)-(7), for any $a \in \mathbb{A}$ with $a^{\prime}=\mathcal{A}(a)>0$ implies

$$
\Pi_{a}\left(a^{\prime}\right) / R+\lambda\left[-u_{c}(c)+\beta B_{a}\left(a^{\prime}\right)\right]=0 .
$$

Suppose $\Pi_{a}\left(a^{\prime}\right)<0$. Since $\lambda=1 / u_{c}>0$, (A.12) implies $u_{c}(c)<\beta B_{a}\left(a^{\prime}\right)$. In contrast, at an interior solution in the non-contractible assets problem (17)-(19) we have $u_{c}\left(c^{N}\right)=\beta B_{a}\left(a^{\prime N}\right)$. Hence, for any such $a$, when assets are contractible, the agent saves strictly less (and consumes more) compared to the non-contractible assets case, that is, $c^{N}<c$ and $a^{\prime N}>a^{\prime}$. The opposite is true if $\Pi_{a}\left(a^{\prime}\right)>0$. Note that in part (i) above, we showed that it not possible that $\Pi_{a}(a)=0$ for all $a \in(0, \mathcal{A}(\bar{a})]$ and so, either $\Pi\left(a^{\prime}\right)<0$ or $\Pi\left(a^{\prime}\right)>0$ for some $a \in(\hat{a}, \bar{a}]$.

We conclude the proof by showing that there exists asset level(s) for which future assets are positive in both the contractible and non-contractible assets problems. Indeed, suppose $a^{\prime N}=0$ for all $a \in(\hat{a}, \bar{a}]$, where by Proposition 2 we know $a^{\prime}>0$. We would then have from (19) that $u_{c}\left(\mathcal{C}^{N}(a)\right)-\beta B_{a}(0) \geq 0$ and from (18) that $u\left(\mathcal{C}^{N}(a)\right)+\beta B(0)=B(a)$, both for all $a \in(\hat{a}, \bar{a}]$. Since $B(a)$ is strictly increasing, the latter implies that $\mathcal{C}^{N}(a)$ is strictly increasing in $a$ and hence the left-hand side of the inequality $u_{c}\left(\mathcal{C}^{N}(a)\right)-\beta B_{a}(0) \geq 0$ is strictly decreasing in $a$. But then it must be that $u_{c}\left(\mathcal{C}^{N}(a)\right)-\beta B_{a}(0)>0$ for all $a \in(\hat{a}, \bar{a})$-i.e., constraint (19) is not binding for those $a$ (its Lagrange multiplier is zero). Hence, for any such $a$, the solution to problem (17)-(19) must be the same as the solution to problem (17)-(18). However, this latter problem is exactly the Markov-perfect problem with contractible assets, (6)-(7). This implies that $\mathcal{A}(a)=\mathcal{A}^{N}(a)=0$ for all $a \in(\hat{a}, \bar{a})$, which contradicts Proposition 2. Therefore, there exists some $a^{*} \in(\hat{a}, \bar{a}]$ for which both $a^{\prime}=\mathcal{A}\left(a^{*}\right)>0$ and $a^{\prime N}=\mathcal{A}^{N}\left(a^{*}\right)>0$

\section{Appendix A.7. Proposition 5}

(i) The first-order condition of problem (22) with respect to assets is $-u_{c}(c)+\beta v_{a}\left(a^{\prime}\right)+\xi=0$, where $\xi \geq 0$ is the Lagrange multiplier associated with the non-negativity constraint on assets. The envelope condition is $v_{a}(a)=r u_{c}(c)$. At an interior solution $\xi=0$, and thus, $u_{c}(c)=\beta r u_{c}\left(c^{\prime}\right)$.

(ii) To show that $\exists \hat{a} \in(0, \bar{a}]$ such that $a^{\prime}=\mathcal{A}(a)=0$ for all $a \in[0, \hat{a}]$ and $a^{\prime}=\mathcal{A}(a)>0$ otherwise, let $a=0$ and guess $a^{\prime}=0$. By Assumption $1,-u_{c}(\bar{y})+\beta r u_{c}(\bar{y})<0$, which, using the first-order and envelope conditions implies $\xi>0$, i.e., $a^{\prime}=0$ is verified since the policy function is unique. Proceed as in Proposition 2 using a continuity argument for $a$ sufficiently close to zero. Naturally, the threshold $\hat{a}$ can be different here.

We now show that the consumption time profile is decreasing for all $a \in(0, \bar{a}]$. Suppose first there is a corner solution, $a^{\prime}=0$. Then, $c=\bar{y}+r a>\bar{y} \geq \bar{y}-\mathcal{A}(0)=c^{\prime}$, i.e., $c^{\prime}<c$. For the interior solution case the result $c^{\prime}<c$ follows from Assumption $1(\beta r<1)$, the strict concavity of $u$ and the Euler equation (23).

Next we show that $\mathcal{C}(a)$ is strictly increasing in $a$ for all $a \in \mathbb{A}$. Rewrite the insurer's problem at assets 
$a$ as:

$$
\begin{aligned}
& \max _{c, a^{\prime} \in \mathbb{A}} u(c)+\beta v\left(a^{\prime}\right) \\
& \text { s.t. } c+a^{\prime}=r a+\bar{y}
\end{aligned}
$$

We will use the following auxiliary Lemma.

Lemma A1. Consider the "utility maximization" problem: $\max _{c_{1}, c_{2}} \tilde{u}\left(c_{1}, c_{2}\right)=\phi^{1}\left(c_{1}\right)+\phi^{2}\left(c_{2}\right)$ subject to $p_{1} c_{1}+p_{2} c_{2}=m$, with $\phi^{1}($.$) and \phi^{2}($.$) strictly increasing and strictly concave and p_{1}, p_{2}>0$. Then, $c_{1}$ and $c_{2}$ are normal goods for a consumer with these preferences and "income" $m$.

Proof of Lemma A1: The fact that additively separable utility with strictly concave sub-components implies normality is well-known (e.g., see Liebhafsky, 1969)

The functions $u$ and $v$ are strictly increasing and strictly concave, thus by Lemma A1 applied to problem (A.13), $c=\mathcal{C}(a)$ and $a^{\prime}=\mathcal{A}(a)$, when not at a corner, are strictly increasing in $a$. This, combined with our previous result that $c^{\prime}=\mathcal{C}\left(a^{\prime}\right)<c=\mathcal{C}(a)$ implies $a^{\prime}=\mathcal{A}(a)<a$ for all $a \in(0, \bar{a}]$. Given these results, property (iii) from Proposition 2 can be shown in analogous way as before. The positive long-run consumption level is $c^{*}=\bar{y}$.

(iii) Suppose the agent is offered $\mathcal{T}(a)=\bar{y}$ as in MPE with free entry by insurers and contractible assets but we allow the agent to choose his own savings, $a^{\prime}$. The first-order condition of the agent's problem given $\mathcal{T}(a)=\bar{y}$ implies $-u_{c}\left(\bar{y}+r a-a^{\prime}\right)+\beta v_{a}\left(a^{\prime}\right)=0$ at an interior solution. Since transfers do not depend on assets, the agent's envelope condition is $v_{a}(a)=r u_{c}\left(\bar{y}+r a-a^{\prime}\right)$ which, plugging in for $v_{a}\left(a^{\prime}\right)$ back into his first-order condition, implies $u_{c}(c)=\beta r u_{c}\left(c^{\prime}\right)$ - the same Euler equation governing asset choice as derived in part (i) where assets are contractible 